\title{
Adiabatic and Nonadiabatic Charge Transport in Li-S Batteries
}

Park, Haesun ; Kumar, Nitin; Melander, Marko; Vegge, Tejs; García Lastra, Juan Maria; Siegel, Donald Jason

Published in:

Chemistry of Materials

Link to article, DOI:

10.1021/acs.chemmater.7b04618

Publication date:

2018

Document Version

Peer reviewed version

Link back to DTU Orbit

Citation (APA):

Park, H., Kumar, N., Melander, M., Vegge, T., García Lastra, J. M., \& Siegel, D. J. (2018). Adiabatic and Nonadiabatic Charge Transport in Li-S Batteries. Chemistry of Materials, 30(3), 915-928.

https://doi.org/10.1021/acs.chemmater.7b04618

\section{General rights}

Copyright and moral rights for the publications made accessible in the public portal are retained by the authors and/or other copyright owners and it is a condition of accessing publications that users recognise and abide by the legal requirements associated with these rights.

- Users may download and print one copy of any publication from the public portal for the purpose of private study or research.

- You may not further distribute the material or use it for any profit-making activity or commercial gain

- You may freely distribute the URL identifying the publication in the public portal

If you believe that this document breaches copyright please contact us providing details, and we will remove access to the work immediately and investigate your claim 


\title{
Adiabatic and Nonadiabatic Charge Transport in Li-S Batteries
}

\author{
Haesun Park, ${ }^{\#, \dagger}$ Nitin Kumar,,${ }^{\#+}$ Marko Melander, ${ }^{\square,}, \#+$ Tejs Vegge, ${ }^{\square}$ Juan Maria Garcia

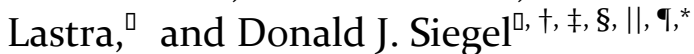 \\ ${ }^{\dagger}$ Mechanical Engineering Department, ${ }^{\ddagger}$ Materials Science \& Engineering, ${ }^{\S}$ Applied Physics Program, ${ }^{\prime}$ Michigan \\ Energy Institute, and ${ }^{\top} J o i n t$ Center for Energy Storage Research, University of Michigan, Ann Arbor, Michigan \\ 48109-2125, United States \\ ${ }^{\square}$ Department of Energy Conversion and Storage, Technical University of Denmark, Fysikvej, Building 309, 280o Kgs \\ Lyngby, Denmark \\ Department of Chemistry, Nanoscience Center, University of Jyväskylä, FI-40o14 Jyväskylä, Finland
}

\begin{abstract}
The insulating nature of the redox end members in Li-S batteries, $\alpha-\mathrm{S}$ and $\mathrm{Li}_{2} \mathrm{~S}$, has the potential to limit the capacity and efficiency of this emerging energy storage system. Nevertheless, the mechanisms responsible for ionic and electronic transport in these materials remain a matter of debate. The present study clarifies these mechanisms - in both the adiabatic and nonadiabatic charge transfer regimes - by employing a combination of hybrid-functional-based and constrained density functional theory calculations. Charge transfer in $\mathrm{Li}_{2} \mathrm{~S}$ is predicted to be adiabatic, and thus is well described by conventional DFT methodologies. In sulfur, however, transitions between $\mathrm{S}_{8}$ rings are nonadiabatic. In this case, conventional DFT overestimates charge transfer rates by up to 2 orders of magnitude. Delocalized holes, and to a lesser extent, localized electron polarons, are predicted to be the most mobile electronic charge carriers in $\alpha-\mathrm{S}_{\text {; }} \mathrm{Li}_{2} \mathrm{~S}$ hole polarons dominate. Although all carriers exhibit extremely low equilibrium concentrations, and thus yield negligible contributions to the conductivity, their mobilities are sufficient to enable the sulfur loading targets necessary for high energy densities. Our results highlight the value of methods capable of capturing nonadiabadicty, such as constrained DFT. These techniques are especially important for molecular crystals such as $\alpha$-S, where longer-range charge transfer events are expected. Combining the present computational results with prior experimental studies, we conclude that low equilibrium carrier concentrations are responsible for sluggish charge transport in $\alpha-\mathrm{S}$ and $\mathrm{Li}_{2} \mathrm{~S}$. Thus, a potential strategy for improving the performance of Li-S batteries is to increase the concentrations of holes in these redox end members.
\end{abstract}

\section{INTRODUCTION}

The lithium-sulfur (Li-S) system is an emerging, "beyond Li-ion" battery chemistry, ${ }^{6-10}$ with a high theoretical energy density $(2,199 \mathrm{Wh} / \mathrm{l})$ and specific energy $(2,567 \mathrm{Wh} / \mathrm{kg})^{10}$, potential for low cost, ${ }^{7}$ and low toxicity. ${ }^{11}$ Unfortunately, these benefits have not yet been fully realized in practical $\mathrm{Li}-$ $S$ cells, largely due to two unmet challenges. These include polysulfide (PS) dissolution/shuttling and the insulating nature of the redox end-members (REM), $\alpha-\mathrm{S}$ and $\mathrm{Li}_{2} \mathrm{~S}{ }^{7}$ PS dissolution has been widely studied; ${ }^{6,7}$ strategies to suppress dissolution include the use of cathode architectures that physically and/or chemically confine S and PS, ${ }^{6,12-16}$ and substitution of conventional electrolytes with solids, or with those that are non-solvating, or sparingly-solvating, for PS. ${ }^{8,17-25}$

Strategies aimed at suppressing PS dissolution are expected to alter electrochemical reactions in the cathode such that they become localized near the surface of the cathode support, resulting in more solid-state-like behavior. ${ }^{8,26}$ Consequently, transport mechanisms involving solid-state $\mathrm{S}$ and $\mathrm{Li}_{2} \mathrm{~S}$ are expected to play an important role in this 'surface mediated' regime. Transport limitations involving both $\mathrm{S}$ and $\mathrm{Li}_{2} \mathrm{~S}$ REM are possible, as both phases are insulators with large bandgaps. ${ }^{27,28}$

Regarding $\mathrm{Li}_{2} \mathrm{~S}$, the formation of this compound during discharge can mimic the behavior of $\mathrm{Li}_{2} \mathrm{O}_{2}$ in $\mathrm{Li} / \mathrm{O}_{2}$ cells. Earlier studies on the $\mathrm{Li} / \mathrm{O}_{2}$ system have shown that the insulating nature of the $\mathrm{Li}_{2} \mathrm{O}_{2}$ discharge product can limit capacity and increase overvoltages during charging. ${ }^{29-36}$ Thus, by limiting PS solubility in Li-S cells one risks trading the PS dissolution problem for a transport problem arising from cathode passivation. Passivation can also be a concern in Li-S cells in the charged state: oxidation of $\mathrm{Li}_{2} \mathrm{~S}$ during charging generates sulfur, which is also a poor electronic conductor. Transport through $\mathrm{S}$ may also be an important consideration in optimizing the initial distribution and loading of $\mathrm{S}$ in the cathode, to maximize $S$ utilization. In total, transport limitations in Li-S cells may pose challenges that are double those of the $\mathrm{Li} / \mathrm{O}_{2}$ system, because in the former chemistry both REM are electronic insulators.

Figure 1 illustrates plausible electronic and ionic transport mechanisms that may occur in the cathode of a $\mathrm{Li}-\mathrm{S}$ cell during discharge or charge. Figure 1(a) demonstrates electronic transport through a sulfur film that covers a 
carbon cathode support. Reduction of $\mathrm{S}_{8}$ to $\mathrm{S}_{\mathrm{x}}{ }^{2-}$ requires charge transport from the current collector to the reaction site on the S surface. This could result in solid-state film growth of $\mathrm{Li}_{2} \mathrm{~S}$ at the reaction site, or the formation of soluble PS that ultimately precipitate as $\mathrm{Li}_{2} \mathrm{~S}$ elsewhere in the cell.

An alternative scenario occurs when $\mathrm{S}$ does not completely cover the carbon support. This creates a threephase boundary between the electrolyte, S, and the support in the fully or partially charged state. ${ }^{37}$ Consequently, facile electronic charge transport would occur (at least initially) through the conductive support, and not through insulating $\mathrm{S}$. However, deposition of $\mathrm{Li}_{2} \mathrm{~S}$ as discharge progresses can ultimately bury the three-phase interface, as depicted in Figure 1(b). In this case, electronic transport through insulating $\mathrm{Li}_{2} \mathrm{~S}$ is necessary during charging.

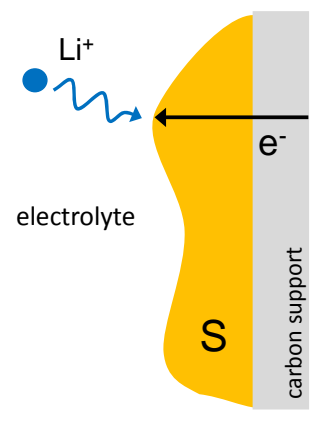

(a) Electronic transport through S during discharge

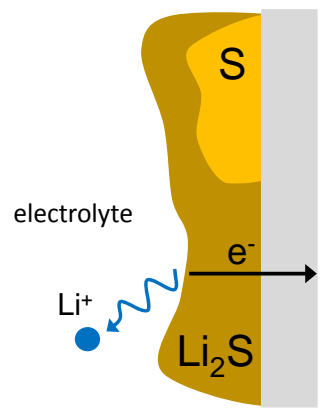

(b) Electronic transport through $\mathrm{Li}_{2} \mathrm{~S}$ during recharge

and ${ }^{41}$.) Finally, Mukherjee et al. reported that the main charge carriers in metastable ${ }^{28} \mathrm{Li}_{2} \mathrm{~S}_{2}$ (based on a hypothetical crystal structure) are hole polarons and negatively charged $\mathrm{Li}$ vacancies. $^{40}$

More generally, nearly all prior studies on charge migration/hopping in cathode materials (typically involving localized electronic carriers such as polarons $)^{38-40}$ have been performed assuming these are adiabatic processes. Within the adiabatic picture, the Born-Oppenheimer approximation is assumed to hold, and it is thus sufficient to map the minimum energy pathway (MEP) along a single, smoothly varying electronic ground state, using, for example, the nudged elastic band (NEB) method (i.e., green curve in Figure 2). ${ }^{42-44}$ The rate constant can then be derived from the energy at the saddle point along the MEP using (harmonic)

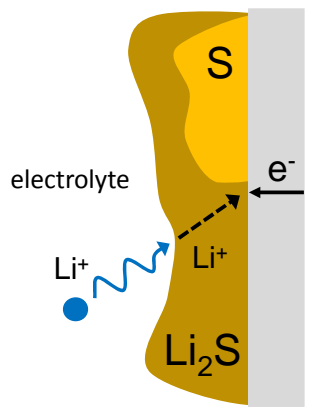

(c) lonic transport through $\mathrm{Li}_{2} \mathrm{~S}$ during discharge

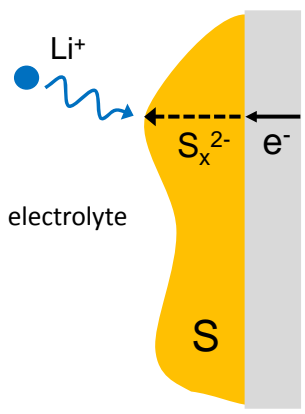

(d) lonic transport through $S$ during discharge

Figure 1. Possible electronic and ionic transport processes in the cathode of a Li-S cell during discharge or charging. Yellow represents sulfur, brown represents $\mathrm{Li}_{2} \mathrm{~S}$, and grey represents the cathode support (assumed to be carbon).

The need for long-range electronic transport can be minimized if rapid ionic transport through $\mathrm{Li}_{2} \mathrm{~S}$ is possible. For example, Figure $1(\mathrm{c})$ shows that $\mathrm{Li}_{2} \mathrm{~S}$ or PS can grow at buried $\mathrm{Li}_{2} \mathrm{~S} / \mathrm{S}$ or $\mathrm{Li}_{2} \mathrm{~S} /$ support interfaces if $\mathrm{Li}_{2} \mathrm{~S}$ conducts $\mathrm{Li}$ ions. Similarly, transport of reduced $\mathrm{S}$ ions, $\mathrm{S}_{\mathrm{x}}{ }^{2}$, through $\mathrm{S}$ (or $\mathrm{Li}_{2} \mathrm{~S}$ ) could allow $\mathrm{Li}_{\mathrm{x}} \mathrm{S}_{\mathrm{y}}$ formation on the surface of these phases, Figure $1(\mathrm{~d})$.

Understanding the transport properties of the $\mathrm{S}$ and $\mathrm{Li}_{2} \mathrm{~S}$ REMs is a prerequisite for the development of rational strategies to improve the capacity, efficiency, and cycle life of Li-S batteries. Nevertheless, transport mechanisms in these compounds have not been widely studied, especially in the case of $\mathrm{S}^{38-41}$ While a few studies exist for $\mathrm{Li}_{2} \mathrm{~S}$, consensus regarding the dominant charge-carrying species has been slow to emerge. For example, Kang et al. reported that the dominant charge carriers in $\mathrm{Li}_{2} \mathrm{~S}$ are negative $\mathrm{Li}$ and positive $S$ vacancies, based on calculations performed at the hybrid functional level of theory. The most likely electronic charge carrier was predicted to be the electron polaron, which localizes on sulfur ions. ${ }^{27}$ In contrast, Mukherjee et al. argue that hole polarons are formed on sulfur ions in $\mathrm{Li}_{2} \mathrm{~S}$ and, together with negative $\mathrm{Li}$ vacancies, serve as lowest energy charge carriers. ${ }^{41}$ Moradabadi and co-workers found that the dominant charge carriers were $\mathrm{Li}$ vacancies and $\mathrm{Li}$ interstitials, based on calculations employing a semi-local functional. ${ }^{39}$ (Li interstitials were not considered in Refs. ${ }^{27}$ transition state theory.

However, charge transfer processes can also be electronically nonadiabatic if the ground and excited states become sufficiently close in energy or cross each other (i.e., a conical intersection) and are thus weakly coupled. Instead of a smooth MEP, the resulting nonadiabatic charge transfer event exhibits an abrupt change and a cusp in the energy along the reaction coordinate, as shown with the dashed curves in Figure 2. In such cases, it is natural to work with diabatic electronic states. These states are not ground states, but retain their chemical and physical character independent of the geometry. Here, the rate constant, $\mathrm{k}_{a \rightarrow b}$, can be expressed using Marcus theory 454647

$$
\mathrm{k}_{a \rightarrow b}=\frac{2 \pi}{\hbar} \frac{\left|H_{b a}\right|^{2}}{\sqrt{4 \pi k_{B} T \lambda}} \exp \left[-\frac{\left(\Delta G^{0}+\lambda\right)^{2}}{4 k_{B} T \lambda}\right],
$$

where $H_{b a}=\langle b|\widehat{H}| a\rangle$ is the coupling constant ( $a$ represents the donor/initial state and $b$ the acceptor/final state), $\Delta G^{0}$ is the reaction free energy between states $a$ and $b$, and $\lambda$ is the reorganization free energy. Nonadiabatic behavior is expected to occur in materials where large hopping distances must be traversed to achieve macroscopic charge transfer. This scenario exists in sulfur for hopping between $\mathrm{S}_{8}$ rings. For such a nonadiabatic reaction, $H_{b a}$ is generally small, and can suppress the hopping rate significantly, Eq. 1. 
The goal of the present study is to elucidate charge transport mechanisms in the $\mathrm{S}$ and $\mathrm{Li}_{2} \mathrm{~S}$ REMs in $\mathrm{Li}-\mathrm{S}$ batteries, while carefully accounting for the (non)adiabatic nature of these processes. The calculated transport rates are

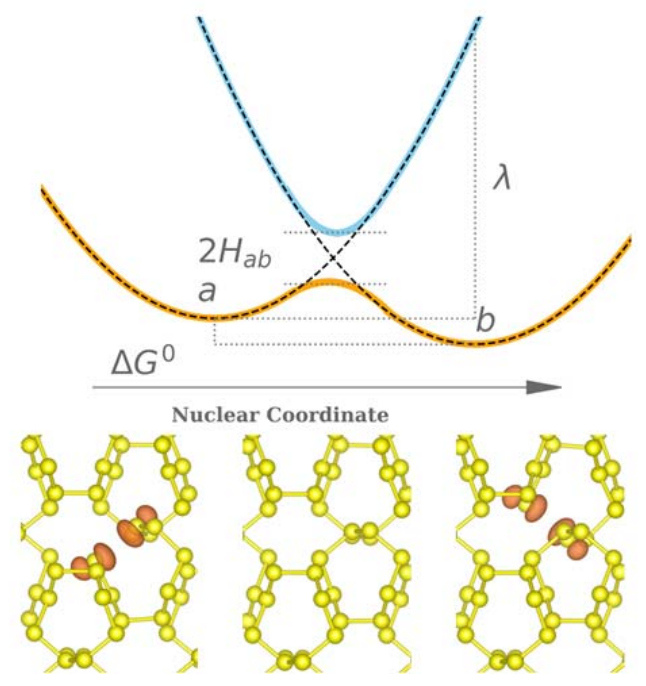

Figure 2. Schematic representation of charge transfer in Marcus theory. The orange (blue) solid line is the adiabatic ground (excited) state while the dashed lines are the two diabatic states describing the initial (a) and final (b) states. $H_{a b}$ is the coupling constant, $\Delta G^{0}$ is the free energy of the reaction, and $\lambda$ is the reorganization

used to estimate the theoretical maximum loadings (i.e., limiting film thicknesses) for $\mathrm{S}$ and $\mathrm{Li}_{2} \mathrm{~S}$ that are consistent with practical discharge rates. More specifically, we separately examine the ionic and electronic conductivity resulting from the formation and migration of ionic and electronic carriers in these two materials. The equilibrium concentrations of several plausible charge carriers and their respective adiabatic mobilities were evaluated at the hybrid functional level of theory, ${ }^{48,49}$ using a tuned fraction of exact exchange. $^{35}$ Subsequently, nonadiabatic effects were examined using the constrained DFT formalism (cDFT). The combination of these approaches allows for a rigorous understanding of how transport phenomena in Li-S cathodes can limit battery performance.

In sulfur, transitions between $\mathrm{S}_{8}$ rings are predicted to be nonadiabatic. Consequently, conventional DFT erroneously overestimates charge transfer rates by up to 2 orders of magnitude, highlighting the importance of going beyond the Born-Oppenheimer approximation. Delocalized holes, and to a lesser extent, localized electron polarons $\left(\mathrm{p}^{-}\right)$, are predicted to be the most mobile electronic charge carriers. All carriers in sulfur exhibit extremely low equilibrium concentrations, and thus yield negligible contributions to the conductivity. Nevertheless, the mobilities of free holes and p are sufficient to enable the sulfur loading targets necessary for high energy densities. Based on the calculated mobility, and assuming a $\mathrm{C} / 5$ discharge rate, we estimate that $\mathrm{p}^{-}$can transit $\mathrm{S}$ films with a thickness of approximately $\sim 100 \mu \mathrm{m}$. The diffusion length for free holes is much larger, $\sim 1 \mathrm{~m}$. These thicknesses exceed the JCESR S loading target of $6 \mathrm{mg} / \mathrm{cm}^{2},{ }^{50,51}$ which correspond to a $\mathrm{S}$ film with an average thickness $30 \mu \mathrm{m}$.

In the case of $\mathrm{Li}_{2} \mathrm{~S}$, positively charged $\mathrm{Li}$ interstitials and negatively charged vacancies are the dominant ionic carriers.
The Li vacancy is most mobile of these carriers, with an activation energy of $0.32 \mathrm{eV}$. The most prevalent electronic carriers are hole polarons; however, these carriers are predicted to have a high formation energy $(1.95 \mathrm{eV})$ and extremely low equilibrium concentrations. Despite its low concentration, the hole polaron is highly mobile, with a maximum migration length of $\sim 40 \mathrm{~mm}$ at a charging rate of $\mathrm{C} / 5$. In contrast, the equivalent migration length for $\mathrm{Li}$ vacancies is much smaller, $\sim 240 \mu \mathrm{m}$. Importantly, the migration lengths for both carriers surpass the projected maximum thickness $(50 \mu \mathrm{m})$ of a $\mathrm{Li}_{2} \mathrm{~S}$ film formed upon discharge (assuming an initial sulfur loading equal to the JCESR target).

\section{METHODOLOGY}

The formation energies and adiabatic mobilities of various charge carriers were evaluated using Density Functional Theory as implemented in the Vienna $a b$ initio Simulation Package (VASP). ${ }^{52,53}$ The simulation cells consisted of the convention al unit cell for $\alpha-S(128$ atoms), and a 96atom supercell constructe d from a $2 \times 2 \times 2$
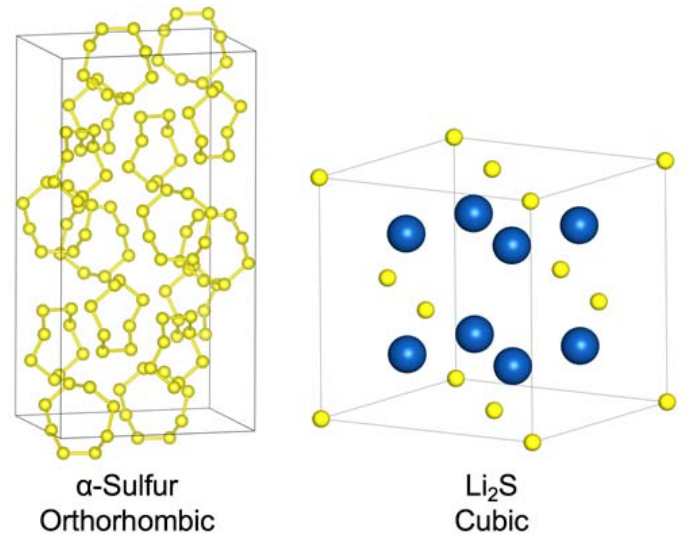

replication of the $\mathrm{Li}_{2} \mathrm{~S}$ unit cell. The unit cells for both materials are shown in Figure 3. Calculations on the bulk properties of $\mathrm{S}$ and $\mathrm{Li}_{2} \mathrm{~S}$ were reported earlier. ${ }^{28}$

The internal degrees of freedom for both supercells were relaxed to a force tolerance of $0.04 \mathrm{eV} / \AA \AA$ ( $\alpha$-S $)$ and $0.01 \mathrm{eV} / \AA$ $\left(\mathrm{Li}_{2} \mathrm{~S}\right)$. The dimensions of the $\alpha-\mathrm{S}$ supercell were previously calculated $^{54}$ using the vdW-DF functional; the following lattice constants were obtained: $\mathrm{a}=10.33 \AA \mathrm{A}=12.76 \AA$, and c $=24.45 \AA$. The present defect calculations are to be performed using hybrid functionals, which do not account for van der Waals interactions between $S_{8}$ rings in $\alpha$-S. Therefore the lattice constants of $\alpha$-S were fixed to the experimental lattice constants, ${ }^{55} \mathrm{a}=10.46 \AA$, b $=12.87 \AA$, and $\mathrm{c}=24.49 \AA$. The lattice constant for $\mathrm{Li}_{2} \mathrm{~S}$ was determined by fitting total energies and volumes to the Murnaghan equation of state $^{56}$ using the $\mathrm{HSE}_{\alpha}$ functional (described below). The calculated lattice constant is $5.68 \AA$ is well matched to the experimental value, ${ }^{57} 5.69 \AA$. K-point sampling was performed at the $\Gamma$-point for $\alpha$-S and using two irreducible k-points for $\mathrm{Li}_{2} \mathrm{~S}$. The projector-augmented wave (PAW) scheme ${ }^{58,59}$ was used to treat core-valence electron interactions. All calculations were spin-polarized with planewave cutoff energies of $450 \mathrm{eV}(\alpha-\mathrm{S})$ and $500 \mathrm{eV}\left(\mathrm{Li}_{2} \mathrm{~S}\right)$.

A total of $18(\alpha-S)$ and $24\left(\mathrm{Li}_{2} \mathrm{~S}\right)$ distinct defects were studied. These defects were considered both as charged and neutral species, and were comprised of vacancies, interstitials, and polarons. Formation energies were 
evaluated for all symmetry-distinct sites. For $\mathrm{Li}_{2} \mathrm{~S}$, Frenkel and Schottky defects were also investigated.

Earlier studies have shown that self-interaction errors present in semi-local functionals can negatively impact the accuracy of these methods when applied to defects that are expected to exhibit localized charge distributions, such as polarons. ${ }^{60-62}$ To minimize these errors, the HeydScuseria-Ernzerhof (HSE) ${ }^{48,49}$ screened hybrid functional was used with the fraction of exact exchange, $\alpha$, set to 0.48 $\left(\mathrm{HSE}_{48}\right)$. Figure $\mathrm{S} 1$ shows the bandgap of $\mathrm{Li}_{2} \mathrm{~S}$ calculated as a function of $\alpha$. At $\alpha=0.48$ the calculated $\mathrm{Li}_{2} \mathrm{~S}$ bandgap (5.28 $\mathrm{eV})$ matches closely the value obtained from many-body $\mathrm{G}_{\mathrm{o}} \mathrm{W}_{\mathrm{o}}(5.27 \mathrm{eV})^{28}$ theory. A similar approach has been used ${ }^{35}$ to examine charge transport in several peroxides and superoxides. ${ }^{35,38,63-65}$

The formation energy $\left(E_{f}\right)$ of a defect $X$ with charge state $\mathrm{q}$ is given by: ${ }^{66,67}$

$$
\begin{aligned}
& \quad E_{f}\left(X^{q}\right)=E_{0}\left(X^{q}\right)-E_{0}(\text { bulk })-\sum_{i} n_{i} \mu_{i}+q \varepsilon_{F}+ \\
& E_{M P 1} .
\end{aligned}
$$

Here $E_{0}\left(X^{q}\right)$ and $E_{0}$ (bulk) correspond to the total energy of the defect-containing and pristine simulation cells, respectively. $n_{i}$ is the number of atoms of the $i^{\text {th }}$ species in the defect cell, and $\mu_{i}$ is that species' chemical potential. $\varepsilon_{F}$ is the Fermi level referenced to the valence band maximum. $\varepsilon_{F}$ is determined by the charge neutrality condition, $\sum_{X^{q}} q C\left(X^{q}\right)=0$, where $C\left(X^{q}\right)$ is the equilibrium concentration of defect $X^{q}$ (defined below). $E_{M P_{1}}$ is the Makov-Payne finite-size correction, which was evaluated using dielectric constants of $7.9\left(\mathrm{Li}_{2} \mathrm{~S}\right)$ and $4.0\left(\mathrm{~S}_{8}\right),{ }^{68}$ and which is applied to supercells containing charged defects. ${ }^{66,69}$ The chemical potential of $\mathrm{S}, \mu_{S}$, is given by the energy per atom of bulk $\alpha-\mathrm{S}$. The chemical potential of $\mathrm{Li}\left(\mu_{L i}\right)$ in the cathode is given by: $\mu_{L i}(L i)=\mu_{L i}(\mathrm{BCC} \mathrm{Li})-e E$, where
$\mu_{L i}(\mathrm{BCC} \mathrm{Li})$ is the energy per atom of bulk (BCC) $\mathrm{Li}$, and $\mathrm{E}=$ 2.3 $\mathrm{V}$ is the theoretical cell voltage of a Li-S cell. ${ }^{35,70}$

The equilibrium concentration $C$ of a defect $X^{q}$ is given by $C\left(X^{q}\right)=D_{X} e^{-E_{f}\left(X^{q}\right) / k_{B} T}$, where $D_{X}$ is the number of equivalent sites per unit volume. ${ }^{67}$ The mobility $(\mu)$ and the diffusion coefficient $(D)$ for a given carrier are given by:

$$
\mu=\frac{v q a^{2}}{k_{B} T} e^{-\frac{E_{b}}{k_{B} T}} \text { and } D=\frac{\mu k_{B} T}{q}=v a^{2} e^{-\frac{E_{b}}{k_{B} T}}(3)
$$

where $v$ is the hopping attempt frequency ${ }^{35}\left(10^{13} \mathrm{~s}^{-1}\right)$ and $a$ is the hopping distance. In the adiabatic approximation, the migration barriers $\left(E_{b}\right)$ of defects are obtained using the climbing image NEB (CI-NEB) method. ${ }^{22-44}$ Due to electron delocalization $^{71}$ errors, semi-local functionals such as the GGA cannot accurately estimate the hopping barrier of localized electronic species, such as polarons. ${ }^{35}$ Hence, we have used the $\mathrm{HSE}_{48}$ functional ${ }^{35,48}$ for computing migration barriers. Finally, defect concentration and mobility are used to estimate the equilibrium conductivity, $\sigma$, associated with a specific defect species, using $\sigma=q C \mu$.

To account for possible nonadiabadic effects in charge transfer reactions, polaron hopping was also studied using constrained DFT $(\mathrm{cDFT})^{72,73}$ as implemented ${ }^{74}$ in the GPAW code, ${ }^{75,76}$ using the $\mathrm{PBE}^{77}$ exchange correlation functional (hybrid functionals have not been implemented in GPAW) and a grid basis with a spacing of $0.16 \AA$. As previously mentioned, GGA functionals over-delocalize charge due to self-interaction errors. However, in cDFT, charge and spin constraints are used to obtain strictly localized charge and magnetization. The cDFT energy is the written as a sum of the usual Kohn-Sham energy, plus a penalty term:

$$
F\left[n,\left\{V_{c}\right\}\right]=E_{K S}[n]+\sum_{i, s} V_{i}\left[\int d \boldsymbol{r} w_{i}^{S}(\boldsymbol{r}) n^{s}(\boldsymbol{r})-N_{i}\right]
$$


where $n$ is electron density, $i$ is an index specifying the constrained region, s refers to spin, $N_{i}$ is the specified charge/spin to be localized in the chosen initial/final state, and $V_{i}$ is the Lagrange multiplier determining the strength of

$$
\mathrm{k}_{a \rightarrow b}=v_{n} \kappa \exp \left[-\frac{\left(\Delta G^{0}+\lambda\right)^{2}}{4 k_{B} T \lambda}+\frac{\Delta}{k_{B} T}\right]
$$

Here $v_{n}$ is the vibrational frequency (taken here as $10^{13} \mathrm{~s}^{-1}$ )

Table 1 Calculated defect formation energies $\left(E_{f}\right)$, equilibrium concentrations $(C)$, hopping barriers $\left(E_{b}\right)$, reorganization energies $(\lambda)$, coupling integrals $\left(H_{a b}\right)$, electronic transmission coefficients $(\kappa)$, rate constants $(k)$, mobilities $(\mu)$, and conductivities $(\sigma)$ for $\alpha-S$ and $\mathrm{Li}_{2} S$. Prior calculations on $\mathrm{Li}_{2} \mathrm{O}_{2}(\operatorname{Ref}$. 30$)$ and $\mathrm{Na}_{2} \mathrm{O}_{2}(\operatorname{Ref}$. 6o) are also shown for comparison.

\begin{tabular}{|c|c|c|c|c|c|c|c|c|c|c|}
\hline Defect Type & $\begin{array}{c}E_{f} \\
(e V)\end{array}$ & $\begin{array}{c}\mathrm{C} \\
\left(\mathrm{cm}^{-3}\right)\end{array}$ & $\begin{array}{c}\text { Adiabatic } \\
\mathrm{E}_{\mathrm{b}}(\mathrm{eV})\end{array}$ & $\begin{array}{c}\text { Diabatic } \\
E_{b}(e V)\end{array}$ & $\begin{array}{c}\lambda \\
(\mathrm{eV})\end{array}$ & $\begin{array}{l}\mathrm{H}_{\mathrm{ba}} \\
(\mathrm{eV})\end{array}$ & $\kappa$ & $\underset{\left(s^{-1}\right)}{k}$ & $\begin{array}{c}\mu \\
\left(\mathrm{cm}^{2} / \mathrm{V} / \mathrm{s}\right)\end{array}$ & $\begin{array}{c}\sigma \\
(\mathrm{S} / \mathrm{cm})\end{array}$ \\
\hline \multicolumn{11}{|c|}{$\alpha-S$} \\
\hline $\mathrm{p}^{-}$(Intra-ring) & \multirow{4}{*}{2.36} & \multirow{3}{*}{$9 \times 10^{-18}$} & 0.11 & 0.07 & 0.45 & 0.19 & 1.0 & $2 \times 10^{11}$ & $3 \times 10^{-3}$ & $4 \times 10^{-39}$ \\
\hline $\mathrm{p}^{-}$(Inter-ring) & & & 0.42 & 0.38 & 1.54 & $1 \times 10^{-3}$ & 0.012 & $4 \times 10^{4}$ & $2 \times 10^{-7}$ & $3 \times 10^{-43}$ \\
\hline $\begin{array}{c}\mathrm{p}^{+} \text {(Intra- } \\
\text { ring })^{\dagger}\end{array}$ & & & & $0.00^{\dagger}$ & 0.18 & 0.09 & & & & \\
\hline $\begin{array}{c}\mathrm{p}^{+}(\text {Inter- } \\
\text { ring })^{\dagger}\end{array}$ & & & & 0.023 & 0.09 & $1 \times 10^{-5}$ & $9 \times 10^{-6}$ & $4 \times 10^{6}$ & & \\
\hline $\begin{array}{c}\text { Delocalized } \\
\text { hole }\end{array}$ & & & & & & & & & $1-10^{\ddagger}$ & $5 \times 10^{-19}$ \\
\hline $\mathrm{S}_{7}^{-}$ & 2.23 & $2 \times 10^{-15}$ & 0.55 & & & & & $5 \times 10^{3}$ & $3 \times 10^{-10}$ & $7 \times 10^{-44}$ \\
\hline $\mathrm{S}_{9}^{+}$ & 2.23 & $3 \times 10^{-17}$ & 1.62 & & & & & $6 \times 10^{-15}$ & $3 \times 10^{-28}$ & $1 \times 10^{-63}$ \\
\hline $\mathrm{S}_{6}^{+}$ & 2.23 & $6 \times 10^{-16}$ & --- & & & & & --- & --- & --- \\
\hline $\mathrm{S}_{7}^{+}$ & 2.25 & $1 \times 10^{-15}$ & 1.20 & & & & & $7 \times 10^{-8}$ & $3 \times 10^{-21}$ & $5 \times 10^{-55}$ \\
\hline & & & & & $\mathrm{i}_{2} \mathrm{~S}$ & & & & & \\
\hline $\mathrm{p}^{+}$ & 1.95 & $4 \times 10^{-11}$ & 0.08 & 0.03 & 0.53 & 0.14 & 1.0 & $6 \times 10^{10}$ & $2 \times 10^{-1}$ & $2 \times 10^{-30}$ \\
\hline $\mathrm{p}^{-}$ & & & & 0.20 & 1.21 & 0.11 & 1.0 & $4 \times 10^{8}$ & & \\
\hline $\mathrm{V}_{\mathrm{Li}}^{-}$ & 0.84 & $3 \times 10^{8}$ & 0.32 & & & & & $4 \times 10^{7}$ & $1 \times 10^{-6}$ & $6 \times 10^{-17}$ \\
\hline $\mathrm{Li}^{+}$ & 0.84 & $1 \times 10^{8}$ & 0.52 & & & & & $2 \times 10^{4}$ & $1 \times 10^{-9}$ & $1 \times 10^{-20}$ \\
\hline $\mathrm{F}_{\mathrm{Li}}$ & 1.22 & $1 \times 10^{2}$ & & & & & & & & \\
\hline $\mathrm{F}_{\mathrm{S}}$ & 1.45 & $2 \times 10^{-2}$ & & & & & & & & \\
\hline Sch & 2.36 & $1 \times 10^{-17}$ & & & & & & & & \\
\hline & & & & & ${ }_{2} \mathrm{O}_{2}$ & & & & & \\
\hline $\mathrm{p}^{+}$ & 0.95 & $1 \times 10^{7}$ & 0.42 & & & & & $7 \times 10^{3 *}$ & -- & $5 \times 10^{-20}$ \\
\hline $\mathrm{V}_{\mathrm{Li}}^{-}$ & 0.93 & $7 \times 10^{7}$ & 0.36 & & & & & $1 \times 10^{5 *}$ & $6 \times 10^{-9}$ & $9 \times 10^{-19}$ \\
\hline
\end{tabular}

the external potential. Both the Lagrange multiplier and electron density are solved self-consistently. $w_{i}^{S}$ is the weight function specifying the spatial extent of the constrained region. Here, the weight function is partitioned into atomic contributions using Hirschfeld partitioning with Gaussian atomic densities based on scaled covalent radii. This ensures that $w_{i}^{S}$ correctly models localization on both atoms and fragments. The constrained charges are formed by adding (removing) one electron to create an electron (hole) polaron and requiring that the region carries a magnetization of 1 . The convergence criterion for cDFT calculations is o.o1 e. (Additional details regarding cDFT can be found in the literature. ${ }^{72,73,74}$ ) In $\mathrm{Li}_{2} \mathrm{~S}$, charge and spin are constrained on a single sulfur atom, as the polarons in this system are localized on atomic sites. In $\alpha$-S the constraining regions are on two neighboring atoms, accounting for the fact that polarons occupy orbitals between $S$ pairs.

Once the diabatic cDFT states are obtained for the initial and final states of a polaron hopping event, the hopping rate is given by the Landau-Zener equation, which captures both adiabatic and nonadiabatic transitions ${ }^{45,46,47}$ :
$\Delta$ is an adiabadicity correction to the diabatic Marcus barrier (described in the Supporting Information), and $\kappa$ is the electronic transmission coefficient computed from the Landau-Zener transition probability ${ }^{45}$

$$
\begin{gathered}
\kappa=\frac{2 P_{L Z}}{1+P_{L Z}} ; \quad P_{L Z}=1-\exp [-2 \pi \gamma] ; \\
2 \pi \gamma=\frac{\pi^{3 / 2}\left|H_{b a}\right|^{2}}{h v_{n} \sqrt{k_{B} T \lambda}} ; \lambda=E_{a}\left(\boldsymbol{R}_{a}\right)-E_{b}\left(\boldsymbol{R}_{b}\right)
\end{gathered}
$$

For an adiabatic reaction $\gamma \gg 1, \kappa \cong 1$, and Eq. 5 reduces to the commonly used transition state rate. For a nonadiabatic reaction, $\gamma<1$ and $\kappa \ll 1$, in which case the Marcus equation is obtained. $\lambda$ is the reorganization energy, and is computed as the energy difference of the donor state in the final and initial state geometries $\boldsymbol{R}_{a}$ and $\boldsymbol{R}_{b}$, respectively. Computation of the coupling constant $\left(H_{b a}\right)$ is described in the Supporting Information.

\section{RESULTS}


Intrinsic Defects in $\alpha$-S. Figure 4 shows the calculated formation energies for the 18 distinct point defects examined in $\alpha$-S. The slope of the lines corresponds to the charge of the defect; a horizontal line represents the formation energy of a neutral species, while positive (negative) sloped lines refer to defects with a positive (negative) charge. Lines with larger slopes correspond to doubly-charged defects. The charge neutrality condition establishes the position of the Fermi

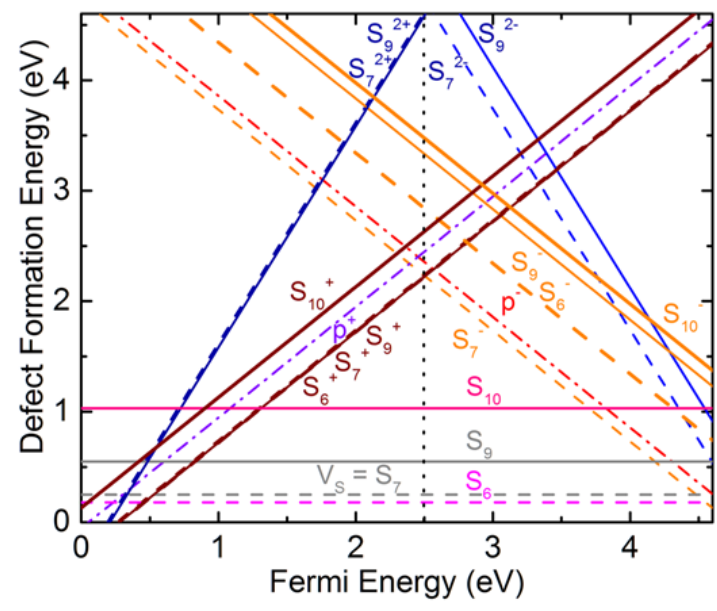

Figure 4. Defect formation energies of interstitials (solid lines), vacancies (dashed lines), and polarons (dash-dot lines) in $\alpha-S$. The vertical dotted line gives the position of tho $r$ r...m: $1 \ldots \ldots$ 1

energy at $2.5 \mathrm{eV}$, and is shown as a vertical dotted line in Figure 4 .

Our calculations indicate that the dominant negative charge carriers in $\alpha-\mathrm{S}$ are $\mathrm{S}$ vacancies $\left(\mathrm{V}_{\mathrm{S}}^{-}\right.$, which appear as an $\mathrm{S}_{7}$ ring with a negative charge, $\left.\mathrm{S}_{7}^{-}\right)$and electron polarons $\left(\mathrm{p}^{-}\right)$, with formation energies of 2.23 and $2.36 \mathrm{eV}$, respectively. A trio of defects comprise the most-prevalent positive localized charge carriers: positive $\mathrm{S}$ interstitials $\left(\mathrm{S}_{\mathrm{i}}^{+}\right.$, equivalent to a positively charged $\mathrm{S}_{9}$ ring, $\left.\mathrm{S}_{9}^{+}\right)$, double $\mathrm{S}$ vacancies $\left(2 \mathrm{~V}_{\mathrm{S}}^{+}=\right.$ $\left.\mathrm{S}_{6}^{+}\right)$, and single $\mathrm{S}$ vacancies $\left(\mathrm{V}_{\mathrm{S}}^{+}=\mathrm{S}_{7}^{+}\right)$. The formation energies for these carriers are 2.23, 2.23, and $2.25 \mathrm{eV}$, respectively. The hole polaron $\left(\mathrm{p}^{+}\right)$has a slightly higher formation energy of $2.45 \mathrm{eV}$. The defect with the lowest formation energy overall is the neutral $\mathrm{S}$ double vacancy $\left(2 \mathrm{~V}_{\mathrm{S}}\right.$ $=\mathrm{S}_{6}$ ), with $E_{\mathrm{f}}=0.18 \mathrm{eV}$. (Delocalized holes also play an important role in transport in $\alpha-S$, and will be discussed separately below.)

We note that the formation energies for all of the charged defects considered are very high (greater than $2.2 \mathrm{eV}$ ); consequently, the concentrations of these carriers under equilibrium conditions will be negligible, as summarized in

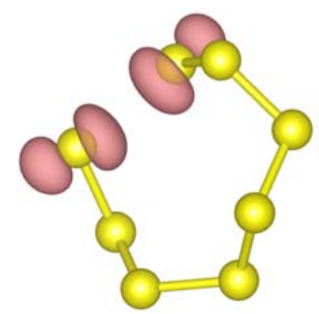

a) Electron polaron, $\mathrm{p}^{-}$ $\Delta \mathrm{E}=-0.42$

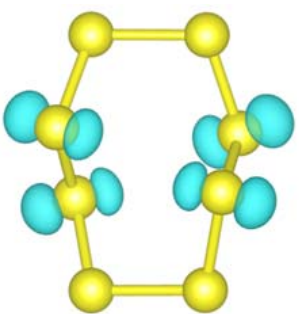

b) Hole polaron, $\mathrm{p}^{+}$

$\Delta \mathrm{E}=+0.48$
Figure 5. Magnetization density for (a) electron $\left(\mathrm{p}^{-}\right)$and (b) hole $\left(\mathrm{p}^{+}\right)$polarons in $\alpha$-S. For clarity, only the $\mathrm{S}_{8}$ molecule where the polaron localizes is shown. The localization energy, $\Delta \mathrm{E}=\mathrm{E}_{\text {localized }}-\mathrm{E}_{\text {delocalized }}$, is shown
Table 1. For example, the highest concentration predicted for a charged defect is $2 \times 10^{-15} \mathrm{~cm}^{-3}$ for $\mathrm{S}_{7}^{-}$. To place this value in context, the concentration of carriers in undoped $\mathrm{Si}$ (which is a poor conductor at room temperature) is 25 orders of magnitude higher. ${ }^{78}$ In contrast, the neutral sulfur vacancy, $V_{S}$, has a relatively high concentration of $2 \times 10^{16} \mathrm{~cm}^{-3}$, yet it will not contribute to conductivity due to its neutrality.

Regarding the properties of polarons, the magnetization densities for both electron and hole polarons in $\alpha$-S are shown in Figure 5. In the case of $\mathrm{p}^{-}$, the extra electron localizes on one of the S-S bonds on a $\mathrm{S}_{8}$ molecule. The shape of the occupied orbital suggests an anti-bonding $\sigma^{*}$ state. The presence of the additional electron results in an elongation of the bond from 2.05 to $2.65 \AA$. A comparison of the energies of localized $\left(\mathrm{p}^{-}\right)$and delocalized electrons shows that localization is preferred by $0.42 \mathrm{eV}$. This is in very good agreement with a prior drift mobility experiment ${ }^{79}$ that determined the $\mathrm{p}^{-}$binding energy to be $0.48 \mathrm{eV}$.

In contrast to the more-localized nature of the electron polaron, the hole polaron $\left(\mathrm{p}^{+}\right)$localizes on two pairs of S-S bonds, Figure $5(\mathrm{~b})$, located on opposite sides of an $\mathrm{S}_{8}$ ring. This results in a slight distortion of the ring, wherein the S-S$\mathrm{S}$ bond angles change from $\sim 107^{\circ}$ to $\sim 102^{\circ}$, with insignificant change in the S-S bond lengths. We find that the $\mathrm{p}^{+}$are unstable with respect to the delocalized state by $0.48 \mathrm{eV}$.

Charge Carrier Mobility in $\alpha-S$. The low equilibrium concentrations of charge carrying defects in $\alpha$-S suggest that even a barrier-less charge hopping process will result in an extremely low conductivity. However, this conclusion assumes that equilibrium concentrations are established. In practice, however, battery operation involves the relatively rapid growth and decomposition of the REM at near-ambient temperatures. Such conditions may generate much higher, non-equilibrium carrier concentrations. This possibility motivates an examination of the mobility of charge carriers in $\alpha$-S.

In the adiabatic limit, mobility can be estimated from the migration energy barriers of the individual defects, calculated using the CI-NEB method. ${ }^{42}$ Figure $6(\mathrm{a})$ shows the minimum energy pathways for the highest-concentration negatively-charged defects in $\alpha$-S. For the ionic carriers, hopping was examined only between adjacent $S_{8}$ rings, i.e. inter-ring hops, as these hoping mechanisms are anticipated to be rate limiting due to their longer hopping distances (compared to intra-ring hops). In the case of $\mathrm{S}_{7}^{-}$, migration occurs with an activation energy of $0.55 \mathrm{eV}$. By way of comparison, this value is 0.1 to $0.2 \mathrm{eV}$ larger than the migration barrier for negative vacancies in $\mathrm{Li}_{2} \mathrm{O}_{2}$ and $\mathrm{Na}_{2} \mathrm{O}_{2}$, Table $1 .{ }^{35,65}$ Hopping of the electron polaron, $\mathrm{p}^{-}$, has a slightly smaller inter-ring hopping barrier of $0.42 \mathrm{eV}$. Polaron hopping was also examined within a single $\mathrm{S}_{8}$ molecule. These intra-ring hops have the lowest activation energy of any carrier considered in $\alpha-\mathrm{S}, 0.11 \mathrm{eV}$. This value agrees well with the experimentally obtained value of $0.167 \mathrm{eV}$ (assuming experiments refer to intra-ring hops). ${ }^{79}$ Nevertheless, we emphasize that hopping via intra-ring processes alone cannot lead to charge transport over macroscopic distances; inter-ring processes constitute the rate-limiting steps.

Figure $6 \mathrm{~b}$ illustrates the adiabatic hopping barriers for the two stable positively charged defects, $\mathrm{S}_{7}^{+}$and $\mathrm{S}_{9}{ }^{+}$. (Migration of $\mathrm{p}^{+}$was not considered due the fact that it is unstable with respect to the delocalized state.) $\mathrm{S}_{9}^{+}$exhibits a relatively high 
activation energy of $1.62 \mathrm{eV}$, while the predicted barrier for $\mathrm{S}_{7}^{+}$is $1.20 \mathrm{eV}$.

The calculations presented above have assumed adiabadicity of the charge transfer events. The validity of this assumption is assessed here using cDFT. All cDFT results are collected in Table 1, and compared with the adiabatic data previously described. We first discuss the behavior of the electron polaron $\left(\mathrm{p}^{-}\right)$in $\alpha-S$, which our $\mathrm{HSE}_{\alpha}$ calculations find to be stable (relative to a delocalized electron). The hopping barriers from cDFT for intra and inter ring electron polaron hops are 0.07 and $0.38 \mathrm{eV}$, respectively. As can be seen in Table 1, the agreement between HSE-NEB and cDFTMarcus barriers is very good - the barriers predicted by the different methods differ by less than $40 \mathrm{meV}$.

Although the good agreement in migration barriers for $\mathrm{p}$ might suggest that all hops can be characterized as adiabatic, the value of the transmission coefficient, $\kappa$, suggests otherwise. More specifically, only the intra-ring process has a $\kappa$ value of 1 , which is indicative of adiabatic behavior. In contrast, the transmission coefficient for the inter-ring hop is very small $(\kappa=0.012)$, indicating that diabatic effects are important. The nonadiabadicity of inter ring $\mathrm{p}^{-}$transfer is due to the weak coupling between the initial and final states $\left(\mathrm{H}_{\mathrm{ba}}=1 \times 10^{-3}\right)$, which is consistent with the relatively large distance, $3.4 \AA$, between them. In total, inter-ring electron transfer exhibits a diabatic hopping rate constant $\mathrm{k}$ of $4 \times 10^{4} \mathrm{~s}^{-}$ ${ }^{1}$. This value is approximately 2 orders of magnitude smaller than the equivalent adiabatic rate constant. The values reported in Table 1 for the hopping rate, mobility, and conductivity of electron polarons account for the nonadiabaticity described above. The mobility estimated for intra-ring $\mathrm{p}^{-}$migration is $3 \times 10^{-3} \mathrm{~cm}^{2} / \mathrm{V} / \mathrm{s}$, which is in close agreement with that determined in drift mobility experiments $\left(6.2 \times 10^{-4} \mathrm{~cm}^{2} / \mathrm{V} / \mathrm{s}\right)$, assuming the same hopping mechanism is probed experimentallly. ${ }^{79}$

Regarding hole polarons, the cDFT calculations confirm the results from the previously-described hybrid functional calculations, indicating that $\mathrm{p}^{+}$are unstable. For example, the barrier for an intra-ring hop of $\mathrm{p}^{+}$vanishes when charge is constrained to reside on two neighboring S-atoms. This suggests that positive charge is delocalized at least across a single sulfur ring. However, hole polaron transfer to an adjacent ring is predicted to be slow and clearly nonadiabatic, as inferred from the transmission coefficient value $\kappa \sim 10^{-6}$. Nevertheless, the inter-ring hole polaron transfer barrier of $23 \mathrm{meV}$ is smaller than thermal energy at room temperature and therefore the hole polaron in $\alpha-S$ is expected to delocalize over distances larger than that of a single $\mathrm{S}_{8}$ ring. This hypothesis was confirmed using nonadiabatic Ehrenfest nuclear-electron dynamics, as detailed in the Supporting Information. As shown in Figure S2, the Ehrenfest dynamics simulation predicts that a localized hole on a single $\mathrm{S}_{8}$ ring becomes delocalized on $\mathrm{p}$ type orbitals across all $128 \mathrm{~S}$ atoms in the simulation cell. This delocalization occurs within $10 \mathrm{fs}$, and without changes to the S-S bond lengths, pointing to a band conduction mechanism for hole transport, rather than localized (polaronic) hopping.

Conductivity in the band-like regime is mainly governed by the scattering of charge carriers by impurities and vibrational modes of the crystal, which in turn determine their mean free path (or, equivalently, the mean free time, $\tau$ ); thus, transition state and Marcus theory, are not applicable. In principle, the impact of impurities on the mean free path could be obtained through ab initio methods, by combining the non-equilibrium Green's function (NEGF) formalism with DFT. ${ }^{80}$ Within this method, it is possible to derive the transmission coefficient due to scattering from each impurity type. Combining this data with the impurity concentration yields the contribution of the impurities to the mean free path. Although the presence of impurities in molecular crystals can reduce carrier mobility significantly, (e.g. up to two orders of magnitude in pentacene ${ }^{81}$ ), the mean free path in these systems is most strongly influenced by scattering from the vibrational modes of the crystal, in particular from the acoustic modes. ${ }^{4,82}$ These low frequency modes are derived from intermolecular forces. In principle, scattering from vibrations could be characterized through an exhaustive analysis of the electron-phonon coupling, ${ }^{82,83}$ however, accurately describing weak intermolecular interactions (and their associated low frequency modes), remains a significant challenge, and is not practical at present. Recombination of carriers is another feature that complicates the study of mobility in $\alpha$-S. As we discuss below, holes move much faster than electrons in $\alpha$-S (due to band conduction of the former vs. hopping for the latter), making the impact of their recombination to the conductivity non-negligible. ${ }^{1}$

Conductivity and Diffusion Length in $\alpha$-S. As expected from the extremely low equilibrium concentrations of charged defects, the conductivity of $\alpha$-S arising from hopping mechanisms (which are generally slow processes) is negligible, Table 1. For example, the inter-ring migration of electron polarons, $\mathrm{p}^{-}$, is the process with the highest hopping-like conductivity of those that contribute to longrange transport. Nevertheless, the value calculated for its conductivity, $\sigma=3 \times 10^{-43} \mathrm{~S} / \mathrm{cm}$, is extremely small. Similarly, the highest hopping-type conductivity attributed to positive carriers is also vanishingly-small, and arises from vacancy migration, $\mathrm{V}_{\mathrm{S}}^{+}=\mathrm{S}_{7}^{+}$, with $\sigma=5 \times 10^{-55} \mathrm{~S} / \mathrm{cm}$.

Table 2. Charge carrier diffusion $(L)$ and drift $\left(L_{d}\right)$ lengths (in $\mu \mathrm{m}$ ) for the predominant charge-carrying species in $\alpha-S$ and $\mathrm{Li}_{2} \mathrm{~S}$ as a function of rate.

\begin{tabular}{|c|c|c|c|c|c|c|}
\hline \multirow{2}{*}{ Defect Type } & \multicolumn{2}{|c|}{$\mathrm{C} / 10$} & \multicolumn{2}{|c|}{$\mathrm{C} / 5$} & \multicolumn{2}{|c|}{${ }_{1 C}$} \\
\hline & $\mathrm{L}$ & $\mathrm{L}_{\mathrm{d}}$ & $\mathrm{L}$ & $\mathrm{L}_{\mathrm{d}}$ & $\mathrm{L}$ & $\mathrm{L}_{\mathrm{d}}$ \\
\hline \multicolumn{7}{|c|}{$\alpha-S$} \\
\hline $\mathrm{p}^{-}$(Inter-ring) & 131 & 258 & 93 & 182 & 41 & 82 \\
\hline $\mathrm{S}_{7}^{-}$ & 5 & 10 & 4 & 7 & 2 & 3 \\
\hline $\mathrm{S}_{7}^{+}$ & o & o & o & o & o & o \\
\hline $\mathrm{S}_{9}^{+}$ & o & o & o & o & o & o \\
\hline $\begin{array}{c}\text { Delocalized } \\
\text { holes }\end{array}$ & & $10^{6}$ & & $10^{6}$ & & $10^{5}$ \\
\hline \multicolumn{7}{|c|}{$\mathrm{Li}_{2} \mathrm{~S}$} \\
\hline $\mathrm{V}_{\mathrm{Li}}^{-}$ & 345 & 678 & 244 & 479 & 109 & 214 \\
\hline $\mathrm{Li}^{+}$ & 11 & 22 & 8 & 16 & 4 & 7 \\
\hline
\end{tabular}


However, we have shown that holes in $\alpha$-S are delocalized and thus migrate via a band conduction mechanism, which is faster than any hopping process. As previously mentioned, analysis of the band conductivity for charge carriers in molecular crystals is a non-accessible quantity with current $a b$ initio methods. Conversely, the experimental mobility of holes in $\alpha$-S has been measured through drift experiments, and is in the range $\mu=1-10 \mathrm{~cm}^{2} / \mathrm{V} / \mathrm{s},{ }^{1,3,4,84}$ at $300 \mathrm{~K}$. This value is 7 orders of magnitude larger than for any of the hopping mechanisms considered here. Experiments also show that the hole mobility decays with temperature as $\mu \propto \mathrm{T}^{-\mathrm{n}}$ with $\mathrm{n}$ $=1.6,1.1$, and 1.7 in the [100], [010], and [001] directions, respectively. ${ }^{84}$ This is also consistent with a band conduction model, where the mobility is expected to decay as $\mu \propto \mathrm{T}^{-1.5}$ due to scattering from acoustic modes (for optical modes $\left.\mu \propto \mathrm{T}^{-0.5}\right) .^{85}$

The calculated diffusivities (Table $\mathrm{S}_{1}$ ) and mobilities were used to establish the migration lengths for charge carriers in $\alpha$-S. (In the case of delocalized holes, the experimental mobility was used.) These lengths provide an upper bound for the thickness of a S film (i.e., maximum S loading) that can be traversed by these carriers during cell operation at a given $C$-rate. Assuming a uniform film of $\alpha$-S with density $\rho_{\alpha-}$ $\mathrm{s}=2 \mathrm{~g} / \mathrm{cm}^{3}$, a loading target ${ }^{51}$ of $6 \mathrm{mg} / \mathrm{cm}^{2}$ results in a $S$ film with a thickness of $30 \mu \mathrm{m}$. Based on the mobility data shown in Table 1, and assuming C-rates (discharge durations) of ${ }_{1} \mathrm{C}$ (360o sec), C/5 (180oo sec), and C/10 (360oo sec), Table 2 summarizes the maximum diffusion lengths, $L=\sqrt{D t}$, for the dominant hopping-type carriers in $\alpha$-S: $\mathrm{p}^{-}, \mathrm{S}_{7}^{-}, \mathrm{S}_{7}^{+}$, and $\mathrm{S}_{9}^{+}$. The maximum diffusion lengths for $\mathrm{p}^{-}$are 131,93 , and $41 \mu \mathrm{m}$ for rates of $\mathrm{C} / 10, \mathrm{C} / 5$ and $1 \mathrm{C}$, respectively, assuming interring hopping between $\mathrm{S}_{8}$ molecules. This suggests that polarons in $\alpha$-S have sufficient mobility to traverse typical film distances corresponding to the JCESR target $\mathrm{S}$ loading. In contrast, the diffusion lengths of the dominant ionic species $\left(\mathrm{S}_{7}^{-}, \mathrm{S}_{7}^{+}\right.$, and $\mathrm{S}_{9}^{+)}$are all well below the thickness target.

The estimates for the diffusion lengths provided above are based on diffusion in the presence of a concentration gradient. Alternatively, in the presence of an electric field, the drift length, $L_{d}$, of a charged species is a more relevant measure of a charge carrier's typical transport length. $L_{d}$ is given in terms of the mobility, $\mu: L_{d}=\sqrt{\mu V t}$. Here $\mathrm{V}$ is the voltage drop across the $\mathrm{S}$ film, for which we adopt $0.1 \mathrm{~V}$ as a plausible value, and $t$ is the time. With these assumptions, our calculations reveal the drift lengths of the hopping-type carriers are roughly double their diffusion lengths, Table 2. Despite this increase, only $\mathrm{p}^{-}$have sufficient mobility to transit the targeted film thickness. Application of the drift length analysis to delocalized holes in $\alpha-S$, Table 2 , shows that the high mobility of these carriers results in extremely long drift lengths, on the order of 0.1 to $1 \mathrm{~m}$.

The preceding discussion indicates that the mobility of delocalized holes and $\mathrm{p}^{-}$are sufficient to enable high $\mathrm{S}$ loadings (and high capacity) in Li-S cells. Nevertheless, the experimental conductivity of $\alpha-S$ has been reported to be extremely small, $\sim 5 \times 10^{-19} \mathrm{~S} / \mathrm{cm} .^{5}$ Taken together, these data sufficient mobility, but poor conductivity - point to low carrier concentrations as the primary obstacle to effective charge transport in $\alpha-S$. (For example, the large bandgap for $\alpha-S$ suggests that the concentration of free holes at room temperature will also be very small. ${ }^{28}$ ) In an earlier study, we

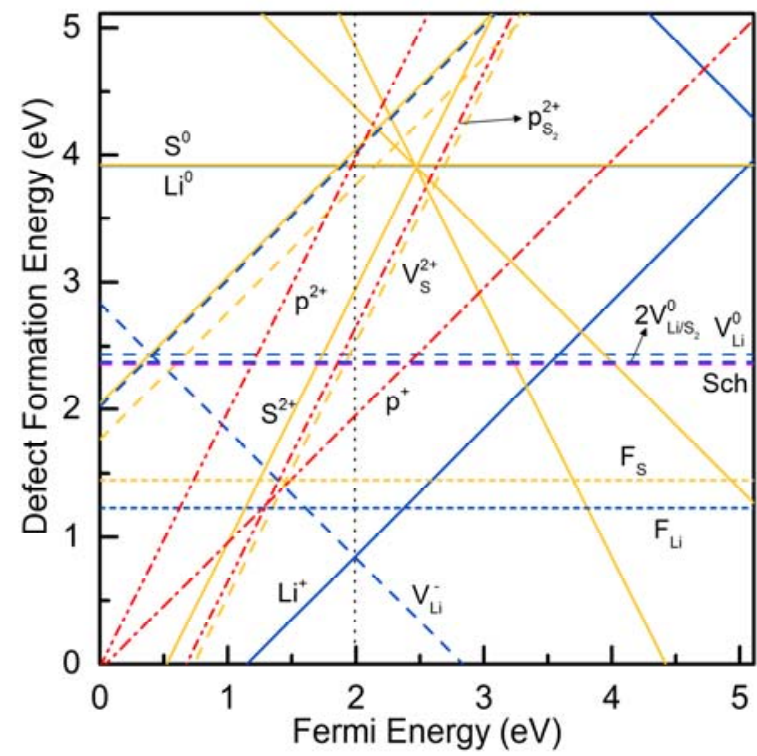

Figure 7. Calculated formation energies for defects in $\mathrm{Li}_{2} \mathrm{~S}$. Yellow lines represent ionic defects on the $\mathrm{S}$ sublattice, blue lines correspond to ionic defects on the $\mathrm{Li}$ sublattice, and red lines refer to polarons. Solid lines are interstitials, while dashed lines represent vacancies. Frenkel defects are identified with the symbols $\mathrm{F}_{\mathrm{S}}$ and $\mathrm{F}_{\mathrm{Li}}$; Schottky defects are identified with magenta lines and the symbol 'Sch.' The vertical dotted line indicates the

explored electrical conductivity limitations in $\mathrm{Li}_{2} \mathrm{O}_{2}$ in the related $\mathrm{Li}_{-} \mathrm{O}_{2}$ system. There, a cathode conductivity of $10^{-11}$ $\mathrm{S} / \mathrm{cm}$ was suggested as a target for achieving efficient operation. ${ }^{35}$ Adopting that value here, and using the calculated mobility for $\mathrm{p}^{-}$, a carrier concentration of $10^{15} \mathrm{~cm}^{-3}$ is required to achieve the conductivity target. This concentration is 33 orders of magnitude larger than the equilibrium $\mathrm{p}^{-}$concentration listed in Table 1 . Similarly, for delocalized holes, the experimental conductivity and mobility data suggest that an increase in carrier concentration of approximately 8 orders of magnitude is required. Thus, strategies for improving transport in $\alpha$-S should target increasing the concentrations of free holes and/or electron polarons.

Intrinsic Defects in $\mathbf{L i}_{2} \mathrm{~S}$. Figure 7 shows defect formation energies in $\mathrm{Li}_{2} \mathrm{~S}$. The predominant defect species, i.e. those having the lowest formation energies, are the negatively charged Lithium vacancy $\left(\mathrm{V}_{\mathrm{Li}}{ }^{-}\right)$, and the positively charged Lithium interstitial $\left(\mathrm{Li}^{+}\right)$, both having formation energies of $0.84 \mathrm{eV}$. The corresponding equilibrium concentrations are $3 \times 10^{8}$ and $1 \times 10^{8} \mathrm{~cm}^{-3}$, respectively; these values are 2 orders of magnitude lower than the carrier concentration in undoped $\mathrm{Si}^{78}$ The lowest-energy neutral defect is the Li Frenkel pair (comprised of a $\mathrm{Li}$ interstitialvacancy pair), with a formation energy of $1.23 \mathrm{eV}$.

Our prediction that $\mathrm{V}_{\mathrm{Li}}{ }^{-}$and $\mathrm{Li}^{+}$are the highestconcentration equilibrium charge carriers in $\mathrm{Li}_{2} \mathrm{~S}$ agrees with the findings of Moradabadi ${ }^{39}$ et al., but differs from the conclusions drawn in two other studies. ${ }^{27,41}$ For example, Kim et $a l^{27}$ found that the dominant carriers are $\mathrm{V}_{\mathrm{Li}}{ }^{-}$and positively two charged $\mathrm{S}$ vacancies $\left(\mathrm{V}_{\mathrm{S}}{ }^{2+}\right)$, with formation energies of $1.31 \mathrm{eV}$. Alternatively, Mukherjee et al. ${ }^{41}$ reported 
$\mathrm{V}_{\mathrm{Li}}{ }^{-}$and hole polarons $\left(\mathrm{p}^{+}\right)$as the predominant species, with formation energies $(1.40 \mathrm{OVV})$ similar to that of Kim et al. These differences can be explained by the omission of interstitial defects in these earlier studies. The present calculations reveal that $\mathrm{Li}^{+}$interstitials are the lowest energy positive charge carrier in $\mathrm{Li}_{2} \mathrm{~S}$. Indeed, the removal $\mathrm{Li}^{+}$from our defect diagram (Figure 7) would shift the Fermi energy to a position similar to that reported by these earlier studies (1.3 - $1.4 \mathrm{eV}$ ), reflecting charge balance between $\mathrm{V}_{\mathrm{Li}}{ }^{-}$and $\mathrm{p}^{+} / \mathrm{V}_{\mathrm{S}}{ }^{2+}$. $\left(\mathrm{p}^{+}\right.$and $\mathrm{V}_{\mathrm{S}}{ }^{2+}$ are the same positive carriers reported in Refs. ${ }^{27,41}$.) We emphasize that an accurate accounting of charge carrier concentrations can only be achieved if a comprehensive sampling of formation energies for all relevant defects - including interstitials - is performed.

The possibility for non-equilibrium carrier concentrations induced by rapid growth/dissolution of $\mathrm{Li}_{2} \mathrm{~S}$ during battery cycling prompts us to examine carriers beyond the ionic species described above, i.e., polarons. The stability of electron and hole polarons in $\mathrm{Li}_{2} \mathrm{~S}$ were explored by adding or removing a single electron from the computational cell, and by applying initial lattice distortions consistent with the presence/absence of localized charge on a $S$ ion. For example, the presence of a hole polaron localized on a $S$ ion (resulting in a charge state of $\mathrm{S}^{-}$) will reduce electrostatic attraction with nearest-neighbor Li ions and thereby increase Li-S distances. In contrast, the presence of an electron polaron will result in formation of an $\mathrm{S}^{3-}$ ion, which will more strongly attract adjacent $\mathrm{Li}^{+}$.

Regarding electron polarons, $\mathrm{p}^{-}$, our attempts to localize an additional electron on a nominally $\mathrm{S}^{2-}$ ion were not successful. Several initial lattice distortions were attempted; nevertheless, in all cases the resulting relaxed structure resembled undistorted $\mathrm{Li}_{2} \mathrm{~S}$, with the extra electron delocalized over the entire computational cell. In contrast, hole polarons, $\mathrm{p}^{+}$, do localize on $\mathrm{S}$ ions. The formation energy
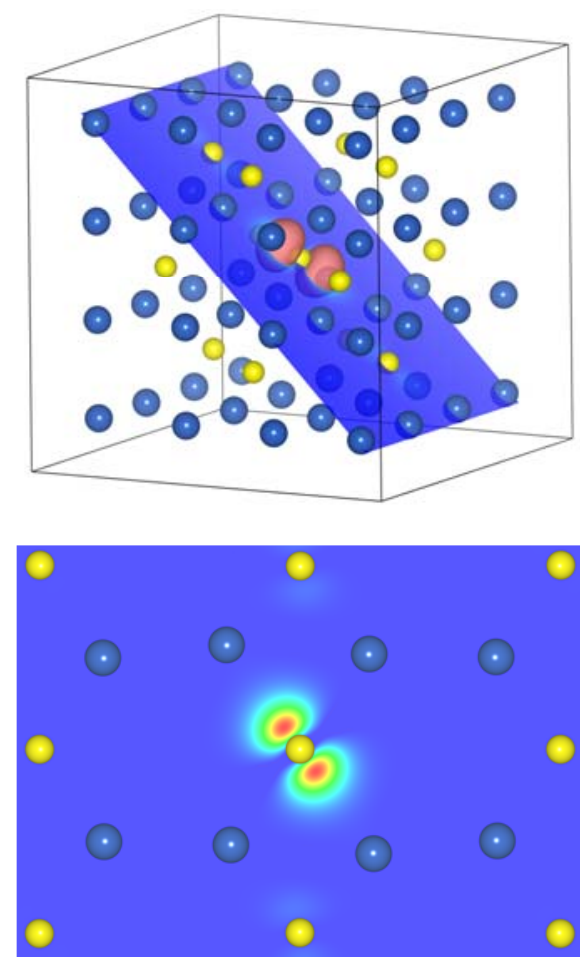

Figure 8. (Top) Magnetization density isosurface for the hole polaron in $\mathrm{Li}_{2} \mathrm{~S}$. (Bottom) Contour plot of the magnetization density in a (110) plane. Red and blue areas represent magnetization densities of $0.06 \mathrm{e} / \mathrm{bohr}^{3}$ and o for $\mathrm{p}^{+}$is high, $1.95 \mathrm{eV}$ (see Table 1 and Figure 7), with a correspondingly low equilibrium concentration of $4 \times 10^{-11} \mathrm{~cm}$ 3 . Furthermore, the localized hole is more stable than a delocalized hole by $0.07 \mathrm{eV}$. Figure 8 (top) shows the magnetization density isosurface for $\mathrm{p}^{+}$, indicating the presence of an unpaired electron localized on a $S$ ion. The spatial distribution of this electron is consistent with that of a 3p-orbital aligned towards a pair of nearest-neighbor Liions, Figure 8(bottom). The Li-ions closest to the localized charge exhibit an enlarged Li-S distance of $2.78 \AA$, which should be compared to $2.46 \AA$ in the absence of $\mathrm{p}^{+}$. (Two metastable $\mathrm{p}^{+}$with distinct local geometries were also identified, and are shown in Figure $\mathrm{S}_{3}$. These configurations are less stable by 13 and $68 \mathrm{meV}$, respectively.) The charge state of an $\mathrm{S}$ anion in the presence of a hole polaron was calculated using a Bader charge analysis. ${ }^{86,87}$ As expected, the total number of valence electrons, 7.2, is significantly smaller in the presence of $\mathrm{p}^{+}$than for a typical $\mathrm{S}^{2-}$ anion, wherein the number of electrons ranges from 7.9 to 8.0.

Our observation of self-trapping of holes on sulfur ions in $\mathrm{Li}_{2} \mathrm{~S}$ agrees with the calculations of Mukherjee et al. ${ }^{41}$ Nevertheless, Ref. ${ }^{27}$ reported a stability trend opposite to those of Mukherjee and the present calculations, with $\mathrm{p}$ being self-trapped and $\mathrm{p}^{+}$being unstable with respect to delocalization. Of course, the formation of $\mathrm{p}^{-}$implies the existence of an $\mathrm{S}^{3-}$ anion, which our intuition suggests would be highly unstable. Moreover, the charge density plot used in Ref. ${ }^{27}$ to substantiate the formation of $\mathrm{S}^{3-}$ does not show a distribution consistent with occupation of a $4 \mathrm{~s}$ orbital, calling into question whether localization of an additional electron has occurred.

Finally, the formation of $\mathrm{S}_{2}{ }^{2-}$ dimers was also investigated by introducing two neutral $\mathrm{Li}$ vacancies, $\mathrm{V}_{\mathrm{Li}}{ }^{\mathrm{o}}$, or by removing two electrons (equivalent to the introduction of two $\mathrm{p}^{+}$) from the simulation cell. These calculations were motivated by the presence of $\mathrm{S}_{2}^{2-}$ dimers in iron pyrite, $\mathrm{FeS}_{2}$, where the S-S distance is $2.16 \AA \AA^{88}$ Dimer formation was induced by initially shortening the distance between adjacent $\mathrm{S}$ ions. Figure $\mathrm{S}_{4}$ illustrates the geometries of these relaxed $\mathrm{S}_{2}{ }^{2-}$ dimers. The resulting $\mathrm{S}_{2}{ }^{2-}$ dimers exhibited S-S distances of 2.10 to $2.13 \AA$. For both scenarios, formation of $\mathrm{S}_{2}{ }^{2-}$ was energetically preferred over the formation of pairs of isolated vacancies or hole polarons. For example, the formation energy of two $\mathrm{V}_{\mathrm{Li}}{ }^{\circ}$ and $\mathrm{S}_{2}{ }^{2-}$ is $2.37 \mathrm{eV}$, while the sum of two single $\mathrm{V}_{\mathrm{Li}}{ }^{\mathrm{o}}$ is much higher, $4.86 \mathrm{eV}$. Similarly, $E_{\mathrm{f}}$ for two $\mathrm{p}^{+}$and an $\mathrm{S}_{2}{ }^{2-}$ is $2.62 \mathrm{eV}$, which is well below that for two $\mathrm{p}^{+}, 3.90 \mathrm{eV}$. These data indicate that it is energetically favorable for $\mathrm{p}^{+}$to localize as pairs on covalently bonded $\mathrm{S}_{2}{ }^{2-}$ dimers. If the charging process involves an initial delithiation step, then the formation of $\mathrm{S}_{2}^{-2}$ dimers would likely be a component of that reaction pathway. 
Charge Carrier Mobility in $\mathrm{Li}_{2} \mathrm{~S}$. The mobilities of $\mathrm{Li}^{+}$, $\mathrm{V}_{\mathrm{Li}}{ }^{-}$, and $\mathrm{p}^{+}$were evaluated in the adiabatic limit using the $\mathrm{HSE}_{\alpha}$ functional and the CI-NEB. Figure 9 shows the minimum energy pathways associated with the migration of

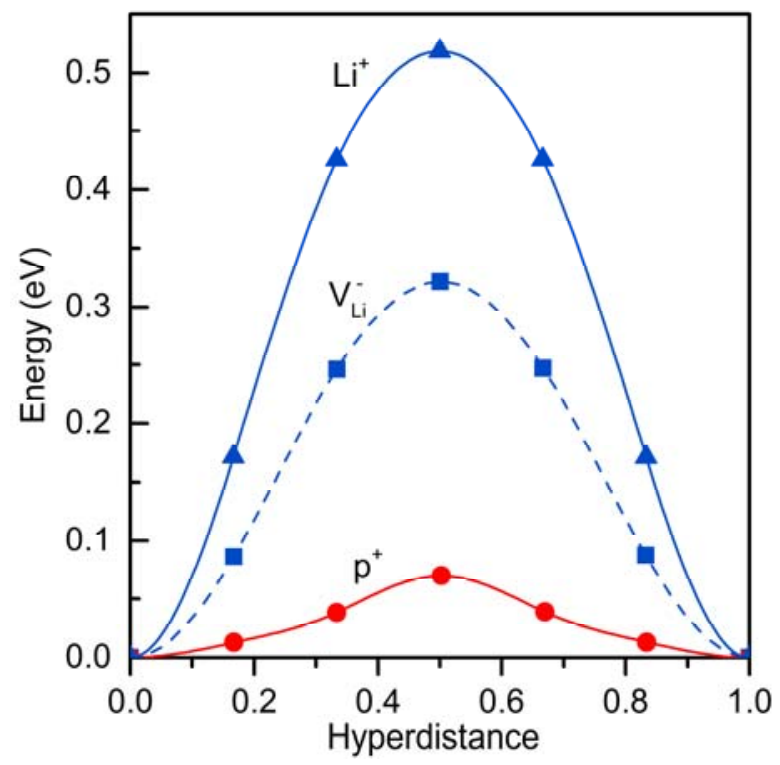

Figure 9. Calculated minimum energy pathways for migration of negative Li vacancies $\left(\mathrm{V}_{\mathrm{Li}}{ }^{-}\right)$, positive lithium interstitials $\left(\mathrm{Li}^{+}\right)$, and hole polarons $\left(\mathrm{p}^{+}\right)$in $\mathrm{Li}_{2} \mathrm{~S}$.

these carriers.

In the case of $\mathrm{Li}^{+}$, two migration mechanisms were examined between neighboring interstitial sites, a simple interstitial hop, and interstitialcy diffusion. The interstitialcy mechanism corresponds to a $\mathrm{Li}^{+}$migrating towards an occupied $\mathrm{Li}$ site, while the ion occupying that site simultaneously hops to a neighboring interstitial position. ${ }^{89}$ Our calculations predict that the interstitialcy mechanism has a much lower energy barrier $\left(E_{a}=0.52 \mathrm{eV}\right)$ than the interstitial process $\left(E_{a}=1.86 \mathrm{eV}\right)$. Based on these activation energies, the corresponding mobilities for these carriers are $6 \times 10^{-10}$ and $2 \times 10^{-32} \mathrm{~cm}^{2} / \mathrm{V} / \mathrm{s}$, respectively. Moradabadi et al. calculated $\mathrm{a} \mathrm{Li}^{+}$interstital migration barrier of $0.47 \mathrm{eV}$ using the PBE-GGA functional. ${ }^{39}$

For $\mathrm{V}_{\mathrm{Li}}{ }^{-}$migration, there is only one symmetry-distinct $\mathrm{Li}$ vacancy site and only one migration pathway between nearest-neighbor sites was considered. The calculated barrier for $\mathrm{V}_{\mathrm{Li}}{ }^{-}$migration, $0.32 \mathrm{eV}$, is $0.2 \mathrm{eV}$ smaller than that for $\mathrm{Li}$ interstitials. This barrier yields a mobility of $1 \times 10^{-6} \mathrm{~cm}^{2} / \mathrm{V} / \mathrm{s}$, which is approximately four orders of magnitude larger than that for $\mathrm{Li}^{+}$mobility. Our calculated energy barrier (evaluated using the $\mathrm{HSE}_{48}$ functional) is in very good agreement with those reported by earlier studies employing the semi-local PBE-GGA functional $\left(\mathrm{E}_{\mathrm{a}}=0.29 \mathrm{eV}\right.$ and 0.27 $\mathrm{eV})^{38,39}$ and the HSEo6 hybrid functional $\left(\mathrm{E}_{\mathrm{a}}=0.29 \mathrm{eV}\right)^{41}$.

For hole polarons, a very small activation energy of 0.08 $\mathrm{eV}$ was calculated for hopping between adjacent $\mathrm{S}$ ions. (The corresponding mobility is $3 \times 10^{-2} \mathrm{~cm}^{2} / \mathrm{V} / \mathrm{s}$.) This value is wellmatched to that of a previous study that predicted a hopping barrier of $0.09 \mathrm{eV} .^{41}$ The predicted hopping barrier is much smaller than that observed for $\mathrm{p}^{+}$migration in $\mathrm{Li}_{2} \mathrm{O}_{2}, \mathrm{Na}_{2} \mathrm{O}_{2}$, and $\mathrm{NaO}_{2}$, where typical barriers are several tenths of an $\mathrm{eV} .^{35,65} \mathrm{On}$ the other hand, the behavior of $\mathrm{p}^{+}$in $\mathrm{Li}_{2} \mathrm{~S}$ is similar to that of hole polarons in $\mathrm{MgO}$, which were reported to have a barrier of only $0.11 \mathrm{eV}$ and mobility of $6 \times 10^{-3}$ $\mathrm{cm}^{2} / \mathrm{V} / \mathrm{s}^{64}$ These data suggest that hole transport in $\mathrm{Li}_{2} \mathrm{~S}$ can be more facile than in the discharge products of, e.g., metalair batteries.

cDFT calculations confirm that both electron and hole polaron transfer reactions are adiabatic $(\kappa=1)$; therefore, the results from HSE-NEB calculations can be used with confidence. Moreover, the barrier for hopping of $\mathrm{p}^{+}$ estimated using cDFT, $0.03 \mathrm{eV}$, differs only by $50 \mathrm{meV}$ from the corresponding $\mathrm{HSE}_{\alpha}$-NEB value described above. Aside from this small difference in the barriers, the rates, mobilities, and conductivities predicted by both approaches (either $\mathrm{HSE}_{\alpha}-\mathrm{NEB}+$ harmonic transition state theory or cDFT + Marcus theory) are equal.

While we were unable to localize the electron polaron using $\mathrm{HSE}_{\alpha}$, localization of $\mathrm{p}^{-}$can be achieved with cDFT. [We emphasize, however, that cDFT does not allow for an estimate of the self-trapping energy of $\mathrm{p}^{-}$, and our earlier conclusion (based on HSE calculations) that electron polarons do not prefer to localize in $\mathrm{Li}_{2} \mathrm{~S}$ remains valid.] Nevertheless, cDFT does provide an opportunity to assess the mobility of $\mathrm{p}^{-}$: these calculations yield a hopping barrier of $0.20 \mathrm{eV}$ and rate constant of $4.3 \times 10^{8} \mathrm{~s}^{-1}$, Table 1. Comparing the barriers and charge transfer rates in Table 1 for hole $\left(6.0 \times 10^{10} \mathrm{~s}^{-1}\right)$ and electron $\left(4.3 \times 10^{8} \mathrm{~s}^{-1}\right)$ polarons, it is apparent that hole polarons are the most mobile electronic carriers in $\mathrm{Li}_{2} \mathrm{~S}$.

Conductivity and Diffusion Length in $\mathrm{Li}_{2} \mathrm{~S}$. Table 2 summarizes the transport properties of the three relevant defects in $\mathrm{Li}_{2} \mathrm{~S}$ identified here - $\mathrm{Li}^{+}, \mathrm{V}_{\mathrm{Li}}{ }^{-}$, and $\mathrm{p}^{+}-$and for comparison revisits the dominant defect chemistry for the peroxides, $\mathrm{Li}_{2} \mathrm{O}_{2}$ and $\mathrm{Na}_{2} \mathrm{O}_{2} \cdot{ }^{35,65}$ The individual contributions of these carriers to the equilibrium ionic and electronic conductivity of $\mathrm{Li}_{2} \mathrm{~S}$ are evaluated in terms of their concentrations and mobilities, $\sigma=q C \mu . \mathrm{V}_{\mathrm{Li}}{ }^{-}$exhibits the highest conductivity of the possible carriers in $\mathrm{Li}_{2} \mathrm{~S}, 6 \times 10^{-17}$ $\mathrm{S} / \mathrm{cm}$. Its conductivity is $2-3$ orders of magnitude larger than $\mathrm{Li}^{+}$interstitials, and for the analogous cation vacancies in $\mathrm{Li}_{2} \mathrm{O}_{2}$ and $\mathrm{Na}_{2} \mathrm{O}_{2}$. This higher conductivity results from a combination of relatively larger concentrations and mobilities of $\mathrm{V}_{\mathrm{Li}}{ }^{-}$in $\mathrm{Li}_{2} \mathrm{~S}$. Nevertheless, a conductivity on the order of $10^{-17} \mathrm{~S} / \mathrm{cm}$ is an extremely low value. In practice, however, the presence of a higher, non-equilibrium carrier concentration may be achieved due to the rapid formation/dissolution of $\mathrm{Li}_{2} \mathrm{~S}$ during battery operation. Measurements of the ionic conductivity of $\mathrm{Li}_{2} \mathrm{~S}$ would be very helpful in identifying these non-equilibrium effects.

Regarding electronic conductivity in $\mathrm{Li}_{2} \mathrm{~S}$, the negligible equilibrium concentration of $\mathrm{p}^{+}, 4 \times 10^{-11} \mathrm{~cm}^{-3}$, results in an extremely low (effectively zero) conductivity, $2 \times 10^{-30} \mathrm{~S} / \mathrm{cm}$. This conductivity is 10 orders of magnitude smaller than in the peroxides, ${ }^{35,65}$ and approaches the value estimated for $\mathrm{MgO}, 3 \times 10^{-36} \cdot{ }^{64}$ Although $\mathrm{p}^{+}$in $\mathrm{Li}_{2} \mathrm{~S}$ have relatively high mobilities, their low concentration offsets the benefits conveyed by these mobilities. Strategies for increasing the carrier concentration could exploit the moderate mobility of $\mathrm{p}^{+}$to improve the electronic conductivity..$^{90}$

We next consider whether the diffusivity and mobility of the dominant charge carriers in $\mathrm{Li}_{2} \mathrm{~S}$ are sufficient to access the full capacity of a Li-S cell. To determine this, a target $\mathrm{S}$ loading of $6 \mathrm{mg} / \mathrm{cm}^{2}$ is adopted (as discussed above), and we 
further assume all $\mathrm{S}$ is reduced during discharge and forms a uniform film of $\mathrm{Li}_{2} \mathrm{~S}$ that covers the cathode support. Based on the density of $\mathrm{Li}_{2} \mathrm{~S}$, such a film will have a thickness of approximately $50 \mu \mathrm{m}$. During charging, charge transport through this film will be necessary; Table 2 summarizes whether the identified carriers have sufficient mobility to transit the film, assuming charging rates of ${ }_{1} C, C / 5$, and $C / 10$. The data reveal that both $\mathrm{V}_{\mathrm{Li}}{ }^{-}$and $\mathrm{p}^{+}$have adequate mobility to cross the film for all rates considered (based on their respective diffusion coefficients). Similarly, the drift length (assuming a voltage drop of $0.1 \mathrm{~V}$ across the $\mathrm{Li}_{2} \mathrm{~S}$ film) is sufficient to accommodate a $50 \mu \mathrm{m}$ film. (The drift length is twice larger than the diffusion length.) In contrast, for all rates considered, the mobility of $\mathrm{Li}^{+}$interstitials is too small to contribute to charge transport across these relatively thick films.

These data indicate that the mobility of $\mathrm{V}_{\mathrm{Li}}{ }^{-}$and $\mathrm{p}^{+}$in $\mathrm{Li}_{2} \mathrm{~S}$ are sufficient to enable high active-material loadings (and high capacity) in Li-S cells. Thus, it is the low equilibrium concentration of carriers that is the primary limitation to effective charge transport. In an earlier study, we explored electrical conductivity limitations in $\mathrm{Li}_{2} \mathrm{O}_{2}$ in the related $\mathrm{Li}-$ $\mathrm{O}_{2}$ system. There, a cathode conductivity of $10^{-11} \mathrm{~S} / \mathrm{cm}$ was suggested as a target for achieving efficient operation. ${ }^{35}$ Adopting that value here, and using the calculated mobility for $\mathrm{p}^{+}$, we determine that a carrier concentration of $10^{8} \mathrm{~cm}^{-3}$ is required to achieve the conductivity target. This concentration is 19 orders of magnitude larger than the equilibrium $\mathrm{p}^{+}$concentration listed in Table 1.

\section{DISCUSSION}

It is worthwhile to reexamine the previous experimental and theoretical studies of the electronic structure and polaron mobility in $\alpha$-S. Firstly, $\alpha-S$ is a molecular solid formed by $\mathrm{S}_{8}$ rings that interact weakly through van der Waals forces. Thus, many of the electronic properties of $\alpha$-S can be roughly understood from the molecular $S_{8}$ vapor phase. Indeed, the photoemission data for $\alpha$-S and molecular $\mathrm{S}_{8}$ are nearly identical, ${ }^{2,91,92}$ apart from the broadening of the lines in the former. As expected in a molecular solid formed by such a small $\mathrm{S}_{8}$ units, the electron-hole interactions are very large. Thus, the molecular excitonic peaks observed in optical absorption experiments in the $\sim 3-5 \mathrm{eV}$ range lie far below the photoemission gap, which is estimated to be close to $8-9 \mathrm{eV} . .^{2}$

Consistent with its molecular nature, the dispersion of the bands in $\alpha-S$ is expected to be very small. Based on optical experiments, Spear and co-workers appraised the valence band bandwidth to be $0.8 \mathrm{o} \mathrm{eV}$, while the conduction band only spanned $0.01 \mathrm{eV} .^{79,91}$ Consequently, the binding energies that stabilize a polaron are negligible for electron polarons, and significantly larger for hole polarons. In fact, free hole polarons (i.e. hole polarons not bonded to any defect) have not been observed in $\alpha$-S, while free electron polarons have been detected with a polaron binding energy of $0.48 \mathrm{eV}^{2,79}$ As mentioned earlier, this binding energy is in good agreement with the value calculated in the present study, $0.42 \mathrm{eV}$.

As described above, our calculations predict that hole polarons in $\mathrm{S}$ cannot be localized on a single bond or $\mathrm{S}_{8}$ ring. This leads us to conclude that hole conduction in sulfur is not polaronic in nature. This conclusion is consistent with experimental conductivity studies, which show that the hole life-time in $\mathrm{S}$ is less than 20 nanoseconds, and that hole transfer is categorized as narrow band conduction rather than hopping. ${ }^{3}$ In the band conduction regime, cDFT coupled with Marcus theory and DFT with transition state theory are inadequate to characterize the conduction mechanism and conductivity. Instead, hole transfer in S should be treated with nonadiabatic quantum dynamics based on the time-dependent Schrödinger equation. This approach naturally includes all possible conduction mechanisms without predefined assumptions. Along these lines, using Ehrenfest dynamics we observed that an initially localized hole polaron (on a single $\mathrm{S}_{8}$ ring) delocalizes within $10 \mathrm{fs}$ onto p-type orbitals spread across all atoms in the simulation cell (without changes in S-S bond lengths). These calculations agree well with the experimental results that show that holes in sulfur are conducted via a band mechanism, rather than via polaron hopping.

Several groups studied electron and hole diffusion in $\alpha-S$ during the 1960's. ${ }^{3,79}$ These studies concluded that at low temperatures (below $300 \mathrm{~K}$ ) the diffusion of holes is controlled by a trapping mechanism, i.e. the holes are trapped at intrinsic defects in the $\alpha$-S crystal and can diffuse with a $\sim 0.22 \mathrm{eV}$ activation barrier. ${ }^{3,79}$ At higher temperatures the trapping diffusion is saturated and the hole transport changes to a lattice scattering regime (i.e. conduction through holes in the valence band). ${ }^{3,79}$ In the present work, we do not consider the transport of polarons bonded to any defect/trap, but instead focus on the diffusion of free polarons. In that context, it is most appropriate to compare our results to the higher temperature experiments. In this latter scenario, our prediction of delocalized holes agrees very well with the band-like conduction observed in experiments.

An analysis of the formation energies of positively charged $S_{x}(x=6-10)$ rings relative to the formation energies of the neutral $S_{x}$ rings (Figure $S_{5}$ ) in $\alpha$-S allows us to identify $S_{9}$ or $\mathrm{S}_{10}$ rings as the low temperature traps observed experimentally ${ }^{3,79}$ (See Supporting Information, Table S2 and Figure S6). In these two rings, localizing a positive charge is more favorable than the delocalized solution by 0.30 and 0.36 $\mathrm{eV}$, respectively. Although the localization energy is larger for $\mathrm{S}_{10}$ rings, most likely the experimental signal of hole traps comes from $S_{9}$ rings, since their formation energy is much lower than that of $S_{10}$ rings. Importantly, the calculated localization energy in $\mathrm{S}_{9}$ rings, $0.30 \mathrm{eV}$ is very close to the experimental activation barrier, $0.22 \mathrm{eV}$. ${ }^{3,79}$ This finding confirms the hypothesis of Gill et al., who claimed that hole traps in $\alpha$-S are formed from native defects. ${ }^{3}$ (Other authors assumed that the hole traps originate from extrinsic defects. ${ }^{4}$ ) Ehrenfest dynamics simulations on an initially localized hole on a $\mathrm{S}_{9}$ ring reveals that the hole primarily remains localized to at least 23 fs (Figure S2). In contrast, a hole on a $\mathrm{S}_{8}$ ring delocalizes completely within $10 \mathrm{fs}$. These direct electron-nuclear dynamics simulations suggest that hole polarons on $\mathrm{S}_{9}$ defects are meta-stable, and can act as polaron traps.

Regarding the migration of electron polarons, two temperature regimes were again identified in experiments. ${ }^{3,79}$ At low temperatures (below $275 \mathrm{~K}$ ), and similar to hole transport, a trapping mechanism with a $\sim 0.40 \mathrm{eV}$ barrier dominates. At higher temperatures, instead of passing to the lattice scatter regime, a free electron polaron hopping with a $\sim 0.20 \mathrm{eV}$ apparent activation barrier was reported as the 
dominant mechanism. ${ }^{3,79}$ Although the experimental barrier of $\sim 0.20 \mathrm{eV}$ is relatively close to the $0.37 \mathrm{eV}$ predicted barrier in our diabatic model, the discrepancy between these values warrants additional discussion. First, the apparent activation energies from experiments are derived from Arrhenius slopes; therefore, any temperature-dependence in the preexponential factor is included in the barrier. This is noteworthy since in Marcus theory the prefactor is (weakly) temperature-dependent. Therefore, both computed and experimental estimates for the effective or apparent barrier from an Arrhenius analysis will include the temperature dependence of the prefactor and the apparent barrier will differ slightly from the barrier values reported in Table 1. Additional factors contributing to this discrepancy are the high concentration of intrinsic defects in the samples $\left(10^{14}\right.$ electron traps per $\mathrm{cm}^{-3}$ in the purest vapor grown samples) and the reported $15 \%$ error in the experimental estimation of the barriers.

To identify the electron polaron traps in $\alpha$-S at low temperature, a similar analysis to the one used for hole polarons was conducted (See Supporting Information). This analysis reveals that $S_{7}$ is the only $S_{x}$ ring in $\alpha$-S where localizing an electron is more favorable than in $S_{8}$ rings (by $0.38 \mathrm{eV}$ ). This energy difference is close to the estimate of $0.40 \mathrm{eV}$ for the activation of electrons at low temperature made by Gill et al. based on measurements on vapor grown samples, corresponding to an electron hop from a $\mathrm{S}_{7}$ to a $\mathrm{S}_{8}$ ring. ${ }^{3,79}$

Regarding $\mathrm{Li}_{2} \mathrm{~S}$, our hybrid functional calculations indicate that hole polarons localized on S ions are stable, while electron polarons are not. Using cDFT, we can nevertheless force the localization of both hole and electron polarons and subsequently evaluate their migration barriers. The values reported in Table 1 indicate that electron polaron transfer in $\mathrm{Li}_{2} \mathrm{~S}$ is much slower than hole transfer, mainly due to the larger reorganization energy required for migration of electron polarons. Based on other recent DFT calculations, hole polaron migration is also predicted to be much faster in $\mathrm{Li}_{2} \mathrm{~S}_{2},{ }^{\circ}$ a possible intermediate product in $\mathrm{Li}-\mathrm{S}$ batteries. Taken together, these experimental and computational studies suggest that hole transport can be fast in $\alpha-\mathrm{S}, \mathrm{Li}_{2} \mathrm{~S}$, and $\mathrm{Li}_{2} \mathrm{~S}_{2}$; this observation implies that the performance of Li-S batteries can be improved (via enhanced electronic transport) by increasing the concentration of holes in these redox end members.

\section{CONCLUSIONS}

Understanding charge transport mechanisms in sulfur and $\mathrm{Li}_{2} \mathrm{~S}$ is a prerequisite for improving the capacity, efficiency, and cycle life of Li-S batteries. In $\alpha$-S, these mechanisms have remained a matter of debate for more than 4 decades. The present study clarifies these mechanisms - in both the adiabatic and nonadiabatic charge transfer regimes - by employing a combination of hybrid-functional-based and constrained density functional theory calculations. The most significant outcomes of these calculations are summarized below.

Charged defects in both $\alpha-\mathrm{S}$ and $\mathrm{Li}_{2} \mathrm{~S}$ are predicted to have high formation energies, resulting in negligible equilibrium carrier concentrations. In contrast, both compounds exhibit high mobilities for a subset of these carriers: in $\alpha$-S, electron polarons and delocalized holes are the most mobile, whereas $\mathrm{Li}$ vacancies and hole polarons dominate in $\mathrm{Li}_{2} \mathrm{~S}$. Importantly, analysis of the drift length for these species reveals that they have sufficient mobility to transit $\alpha-S$ and $\mathrm{Li}_{2} \mathrm{~S}$ films with thicknesses consistent with the JCESR sulfur loading targets. Thus, strategies to improve the conductivity of these materials should focus on increasing carrier concentrations beyond their equilibrium values.

In $\alpha$-S, our calculations demonstrate that electrons can localize into polarons. Polaronic transfer within a single $\mathrm{S}_{8}$ ring is predicted to be fast $\left(10^{11} \mathrm{~s}^{-1}\right)$ and adiabatic. In contrast, polaron hopping between two adjacent $\mathrm{S}_{8}$ rings is nonadiabatic, and much slower $\left(10^{4} \mathrm{~s}^{-1}\right)$. Neglecting nonadiabadicity, as is commonly done in DFT and transition state theory calculations, would overestimate these rate constants (and consequently also the mobility and the conductivity) by two orders of magnitude. This gap highlights the importance of going beyond the BornOppenheimer approximation by including nonadiabatic effects in computational studies of charge transfer kinetics in battery materials. Computational methods that combine cDFT with Marcus theory can treat both adiabatic and nonadiabatic charge transfer on equal footing, and are thus well-suited for these types of investigations.

Although electron polarons are stable in $\alpha$-S, hole polarons are not. Instead, hole transport is expected to follow a band-like mechanism, as suggested by experiments. The formation of delocalized holes in the valence band was confirmed by Ehrenfest dynamics, which show that an initially-localized hole becomes delocalized across the entire computational cell within $10 \mathrm{fs}$, without changes in geometries. Furthermore, our calculations identify $\mathrm{S}_{9}$ and $\mathrm{S}_{7}$ rings as the defects that respectively trap holes and electrons in $\alpha$-S, thus resolving a long-standing question regarding the nature of charge traps in this system.

In $\mathrm{Li}_{2} \mathrm{~S}$, the highest-concentration carriers are ionic species, negative $\mathrm{Li}$ vacancies and positive $\mathrm{Li}$ interstitials. Of these, only vacancies have sufficient mobility to transit $\mathrm{Li}_{2} \mathrm{~S}$ films with thicknesses consistent with the JCESR S loading target. Regarding electronic carriers in $\mathrm{Li}_{2} \mathrm{~S}$, hole polarons are predicted to form, and to be more stable and mobile than electron polarons. Their transport can be categorized as adiabatic, and their mobilities are more than two orders of magnitude larger than for vacancy migration.

\section{ASSOCIATED CONTENT}

\section{Supporting Information}

Band gap of $\mathrm{Li}_{2} \mathrm{~S}$ as a function of exact exchange percentage in HSE, formulas for the adiabadicity correction, calculation of the coupling constant elements, and Ehrenfest dynamics calculations.

\section{AUTHOR INFORMATION}

\section{Corresponding Author}

*E-mail: djsiege@umich.edu. Tel.: +1 (734) 764-48o8.

\section{Author Contributions}

\#These authors contributed equally. 


\section{ACKNOWLEDGMENT}

This work was supported as part of the Joint Center for Energy Storage Research (JCESR), an Energy Innovation Hub funded by the U.S. Department of Energy, Office of Science, and Basic Energy Sciences. MM acknowledges the grant from Alfred Kordelin Foundation as well as computer resources from CSC - IT Center for Science in Espoo, Finland.

\section{REFERENCES}

(1) Dolezalek, F. K.; Spear, W. E. J. Phys. Chem. Solids 1975, 36,

(2) Nitzki, V.; Stössel, W. Phys. Status Solidi B 1970, 39, 309.

(3) Gill, W. D.; Street, G. B.; Macdonald, R. E. J. Phys. Chem. Solids $1967,28,1517$

(4) Adams, A. R.; Spear, W. E. Journal of Physics and Chemistry of Solids 1964, 25, 1113

(5) Neumann, H. Z. Phys. 1927, 45, 717.

(6) Seh, Z. W.; Sun, Y.; Zhang, Q.; Cui, Y. Chem. Soc. Rev. 2016, 45, 5605 .

(7) Pang, Q.; Liang, X.; Kwok, C. Y.; Nazar, L. F. Nat. Energy 2016, 1,16132 .

(8) Cheng, L.; Curtiss, L. A.; Zavadil, K. R.; Gewirth, A. A.; Shao, Y.; Gallagher, K. G. ACS Energy Letters 2016, 1, 503

(9) Manthiram, A.; Chung, S.-H.; Zu, C. Adv. Mater. 2015, 27, 1980.

(10) Bruce, P. G.; Freunberger, S. A.; Hardwick, L. J.; Tarascon, J.M. Nat. Mater. 2012, 11, 19.

(11) Moon, S.; Jung, Y. H.; Jung, W. K.; Jung, D. S.; Choi, J. W.; Kim, D. K. Adv. Mater. $2013,25,6547$.

(12) Ji, X.; Lee, K. T.; Nazar, L. F. Nat. Mater. 2oo9, 8, 500.

(13) Zheng, G.; Yang, Y.; Cha, J. J.; Hong, S. S.; Cui, Y. Nano Lett. 2011, 11, 4462.

(14) Zhang, C.; Wu, H. B.; Yuan, C.; Guo, Z.; Lou, X. W. Angew. Chem. Int. Ed. 2012, 51, 9592.

(15) Zhou, G.; Zhao, Y.; Manthiram, A. Adv. Energy Mater. 2015, 5, 1402263 .

(16) Park, H.; Siegel, D. J. Chem. Mater. 2017, 29, 4932.

(17) Seo, D. M.; Borodin, O.; Han, S.-D.; Boyle, P. D.; Henderson,

W. A. J. Electrochem. Soc. 2012, 159, A1489.

(18) Dokko, K.; Tachikawa, N.; Yamauchi, K.; Tsuchiya, M.; Yamazaki, A.; Takashima, E.; Park, J.-W.; Ueno, K.; Seki, S.; Serizawa, N.; Watanabe, M. J. Electrochem. Soc. 2013, 160, A1304.

(19) Ueno, K.; Park, J.-W.; Yamazaki, A.; Mandai, T.; Tachikawa, N.; Dokko, K.; Watanabe, M. J. Phys. Chem. C 2013, 117, 20509.

(20)Cuisinier, M.; Cabelguen, P. E.; Adams, B. D.; Garsuch, A.; Balasubramanian, M.; Nazar, L. F. Energy Environ. Sci. 2014, 7, 2697.

(21) Yamada, Y.; Yamada, A. J. Electrochem. Soc. 2015, 162, A2406.

(22) Younesi, R.; Veith, G. M.; Johansson, P.; Edstrom, K.; Vegge, T. Energy Environ. Sci. 2015, 8, 1905.

(23) Zhang, S.; Ueno, K.; Dokko, K.; Watanabe, M. Adv. Energy Mater. 2015, 5, 1500117.

(24)Das, S.; Ngene, P.; Norby, P.; Vegge, T.; de Jongh, P. E.; Blanchard, D. J. Electrochem. Soc. 2016, 163, A2029.

(25) Lee, C.-W.; Pang, Q.; Ha, S.; Cheng, L.; Han, S.-D.; Zavadil, K. R.; Gallagher, K. G.; Nazar, L. F.; Balasubramanian, M. ACS Central Science 2017.

(26)Helen, M.; Reddy, M. A.; Diemant, T.; Golla-Schindler, U.; Behm, R. J.; Kaiser, U.; Fichtner, M. 2015, 5, 12146.

(27) Kim, B. S. D.-H.; Lee, M. S. B.; Park, K.-Y.; Kang, K. Chem. Asian J. 2016, 11, 1288.

(28)Park, H.; Koh, H. S.; Siegel, D. J. J. Phys. Chem. C 2015, 119, 4675

(29)Albertus, P.; Girishkumar, G.; McCloskey, B.; Sánchez-Carrera, R. S.; Kozinsky, B.; Christensen, J.; Luntz, A. C. J. Electrochem. Soc. 2011, 158, A343.

(30)Chen, J.; Hummelshøj, J. S.; Thygesen, K. S.; Myrdal, J. S. G.; Nørskov, J. K.; Vegge, T. Catal. Today 2011, Medium: X; Size: p. 2.
(31) Viswanathan, V.; Thygesen, K. S.; Hummelshøj, J. S.; Nørskov, J. K.; Girishkumar, G.; McCloskey, B. D.; Luntz, A. C. J. Chem. Phys. 2011, 135, 214704

(32) Das, S. K.; Xu, S.; Emwas, A.-H.; Lu, Y. Y.; Srivastava, S.; Archer, L. A. Energy Environ. Sci. 2012, 5, 8927.

(33) Lu, Y.-C.; Shao-Horn, Y. J. Phys. Chem. Lett. 2013, 4, 93.

(34) Luntz, A. C.; Viswanathan, V.; Voss, J.; Varley, J. B.; Nørskov, J.

K.; Scheffler, R.; Speidel, A. J. Phys. Chem. Lett. 2013, 4, 3494

(35) Radin, M. D.; Siegel, D. J. Energy Environ. Sci. 2013, 6, 2370.

(36) Viswanathan, V.; Nørskov, J. K.; Speidel, A.; Scheffler, R.; Gowda, S.; Luntz, A. C. J. Phys. Chem. Lett. 2013, 4, 556.

(37) Fan, F. Y.; Carter, W. C.; Chiang, Y.-M. Adv. Mater. 2015, 27, 5203.

(38)Lee, B.; Kim, J.; Yoon, G.; Lim, H.-D.; Choi, I.-S.; Kang, K. Chem. Mater. 2015, 27, 8406.

(39) Moradabadi, A.; Kaghazchi, P. Appl. Phys. Lett. 2016, 108, 213906.

(40) Liu, Z.; Balbuena, P. B.; Mukherjee, P. P. J. Phys. Chem. Lett. 2017, 8, 1324.

(41) Liu, Z.; Balbuena, P. B.; Mukherjee, P. P. J. Phys. Chem. C 2017, 121,17169 .

(42)Henkelman, G.; Jonsson, H. J. Chem. Phys. 2ooo, 113, 9978

(43) Henkelman, G.; Uberuaga, B. P.; Jónsson, H. J. Chem. Phys. 2000, 113, 9901.

(44)Sheppard, D.; Terrell, R.; Henkelman, G. J. Chem. Phys. 2008 $128,134106$.

(45) Kuznetsov, A. M.; Ulstrup, J. 1999.

(46) Marcus, R. A. J. Chem. Phys. 1956, $24,966$.

(47) Marcus, R. A.; Sutin, N. Biochimica et Biophysica Acta, Reviews on Bioenergetics 1985, 811, 265.

(48) Heyd, J.; Scuseria, G. E.; Ernzerhof, M. J. Chem. Phys. 2003, 118,8207 .

(49) Krukau, A. V.; Vydrov, O. A.; Izmaylov, A. F.; Scuseria, G. E.

J. Chem. Phys. 2006, 125, 224106.

(50)Song, M.-K.; Zhang, Y.; Cairns, E. J. Nano Lett. 2o13, 13, 5891.

(51) Eroglu, D.; Zavadil, K. R.; Gallagher, K. G. J. Electrochem. Soc. 2015, 162, A982.

(52) Kresse, G.; Furthmuller, J. Phys. Rev. B 1996, 54, 11169

(53) Kresse, G.; Furthmuller, J. Comput. Mater. Sci. 1996, 6, 15

(54)Adams, A. R.; Gibbons, D. J.; Spear, W. E. Solid State Commun. 1964, 2, 387 .

(55) Rettig, S. J.; Trotter, J. Acta Crystallographica Section C 1987, 43, 2260 .

(56) Murnaghan, F. D. Proc. Natl. Acad. Sci. U.S.A. 1944, 30, 244.

(57) Buehrer, W.; Altorfer, F.; Mesot, J.; Bill, H.; Carron, P.; Smith,

H. G. J. Phys.: Condens. Matter 1991, 3, 1055.

(58) Kresse, G.; Joubert, D. Phys. Rev. B 1999, 59, 1758

(59)Blochl, P. E. Phys. Rev. B 1994, 50, 17953.

(6o) Pacchioni, G. J. Chem. Phys. 2oo8, 128, 182505.

(61) Alkauskas, A.; Broqvist, P.; Pasquarello, A. Phys. Status Solidi B 2011, 248,775

(62)Lambrecht, W. R. L. Phys. Status Solidi B 2011, 248, 1547.

(63) Li, S.; Liu, J.; Liu, B. Chem. Mater. 2017, 29, 2202.

(64) Smith, J. G.; Naruse, J.; Hiramatsu, H.; Siegel, D. J. Chem.

Mater. 2017.

(65)Yang, S.; Siegel, D. J. Chem. Mater. 2015, 27, 3852.

(66) Komsa, H.-P.; Rantala, T. T.; Pasquarello, A. Phys. Rev. B 2012, 86, 045112.

(67)Walle, C. G. V. d.; Neugebauer, J. J. Appl. Phys. 2004, 95, 3851.

(68) In Non-Tetrahedrally Bonded Elements and Binary Compounds I; Madelung, O., Rössler, U., Schulz, M., Eds.; Springer Berlin Heidelberg: Berlin, Heidelberg, 1998, p 1.

(69) Makov, G.; Payne, M. C. Phys. Rev. B 1995, 51, 4014.

(70)Hummelshøj, J. S.; Luntz, A. C.; Nørskov, J. K. J. Chem. Phys. 2013, 138, 034703.

(71) Loftager, S.; García-Lastra, J. M.; Vegge, T. J. Phys. Chem. C 2016, 120, 18355 .

(72) Wu, Q.; Van Voorhis, T. Phys. Rev. A 2005, 72, 024502.

(73) Kaduk, B.; Kowalczyk, T.; Van Voorhis, T. Chem. Rev. 2012, 112, 321.

(74) Melander, M.; Jónsson, E. O.; Mortensen, J. J.; Vegge, T.; García Lastra, J. M. J. Chem. Theory Comput. 2016, 12, 5367. 
(75) Mortensen, J. J.; Hansen, L. B.; Jacobsen, K. W. Phys. Rev. B 2005, 71, 035109.

(76)Enkovaara, J.; Rostgaard, C.; Mortensen, J. J.; Chen, J.; Dułak, M.; Ferrighi, L.; Gavnholt, J.; Glinsvad, C.; Haikola, V.; Hansen, H. A.; Kristoffersen, H. H.; Kuisma, M.; Larsen, A. H.; Lehtovaara, L.; Ljungberg, M.; Lopez-Acevedo, O.; Moses, P. G.; Ojanen, J.; Olsen, T.; Petzold, V.; Romero, N. A.; Stausholm-Møller, J.; Strange, M.; Tritsaris, G. A.; Vanin, M.; Walter, M.; Hammer, B.; Häkkinen, H.; Madsen, G. K. H.; Nieminen, R. M.; Nørskov, J. K.; Puska, M.; Rantala, T. T.; Schiøtz, J.; Thygesen, K. S.; Jacobsen, K. W. J. Phys.: Condens. Matter 2010, 22, 253202.

(77) Perdew, J. P.; Burke, K.; Ernzerhof, M. Phys. Rev. Lett. 1996, 77, 3865 .

(78)Sproul, A. B.; Green, M. A. J. Appl. Phys. 1991, 70, 846.

(79)Gibbons, D. J.; Spear, W. E. J. Phys. Chem. Solids 1966, 27, 1917.

(8o) Garcia-Lastra, J. M.; Mowbray, D. J.; Thygesen, K. S.; Rubio, A.; Jacobsen, K. W. Phys. Rev. B 2010, 81, 10.

(81) Jurchescu, O. D.; Baas, J.; Palstra, T. T. M. Appl. Phys. Lett. 2004, 84, 3061.
(82)Munn, R. W.; Siebrand, W. Discuss. Faraday Soc. 1971, 51, 17. (83) Silbey, R.; Munn, R. W. J. Chem. Phys. 1980, 72, 2763.

(84) Nitzki, V.; Stössel, W. physica status solidi (b) 1970, 39, 309. (85)Ashcroft, N. W.; Mermin, N. D. Solid State Physics; Holt, Rinehart and Winston, 1976.

(86) Henkelman, G.; Arnaldsson, A.; Jónsson, H. Comput. Mater. Sci. 2006, 36, 354 .

(87)Tang, W.; Sanville, E.; Henkelman, G. J. Phys.: Condens. Matter 2009, 21, 084204 .

(88) Stevens, E. D.; DeLucia, M. L.; Coppens, P. Inorg. Chem. 1980, 19, 813 .

(89) Tilley, R. J. D. In Defects in Solids; John Wiley \& Sons, Inc.: 2008, p 205.

(9o) Radin, M. D.; Monroe, C. W.; Siegel, D. J. Chem. Mater. 2015, 27, 839 .

(91) Cook, B. E.; Spear, W. E. J. Phys. Chem. Solids 1969, 30, 1125.

(92)Coppens, P.; Yang, Y. W.; Blessing, R. H.; Copper, W. F.; Larsen, F. K. J. Am. Chem. Soc. 1977, 99, 760.

\section{Table of Contents Graphic}

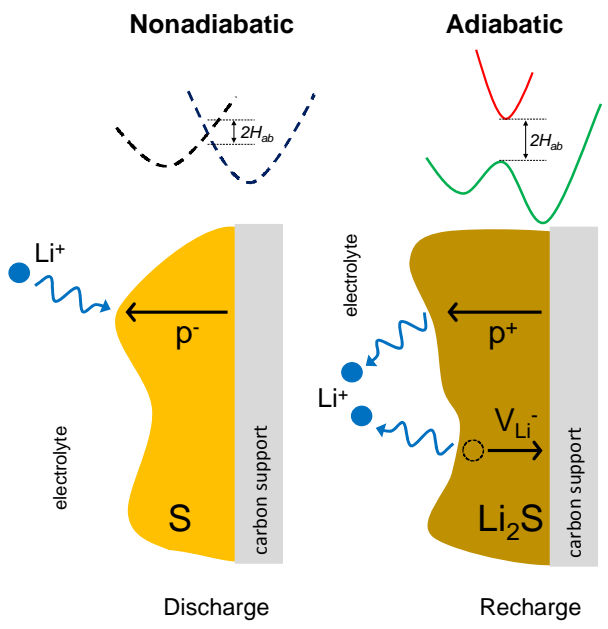




\section{Supporting Information:}

\section{Adiabatic and Nonadiabatic Charge Transport in Li-S Batteries}

Haesun Park ${ }^{\#, \dagger}$, Nitin Kumar ${ }^{\#, \dagger}$, Marko Melander ${ }^{\star} \boldsymbol{\star}^{\star}$, , Tejs Vegge ${ }^{\star}$, Juan Maria Garcia Lastra $^{\star}$, and Donald J. Siegel ${ }^{\star}, \dagger, \ddagger, \S, \|, \uparrow$

${ }^{\dagger}$ Mechanical Engineering Department, ${ }^{\ddagger}$ Materials Science \& Engineering, ${ }^{\S}$ Applied Physics Program, "University of Michigan Energy Institute and "Joint Center for Energy Storage Research, University of Michigan, Ann Arbor, Michigan 48109-2125, United States

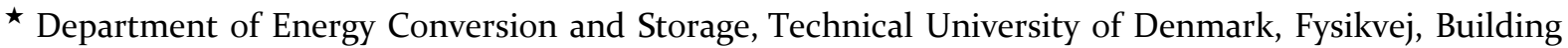
309, 2800 Kgs Lyngby, Denmark

- Department of Chemistry, Nanoscience Center, University of Jyväskylä, FI-40014 Jyväskylä, Finland

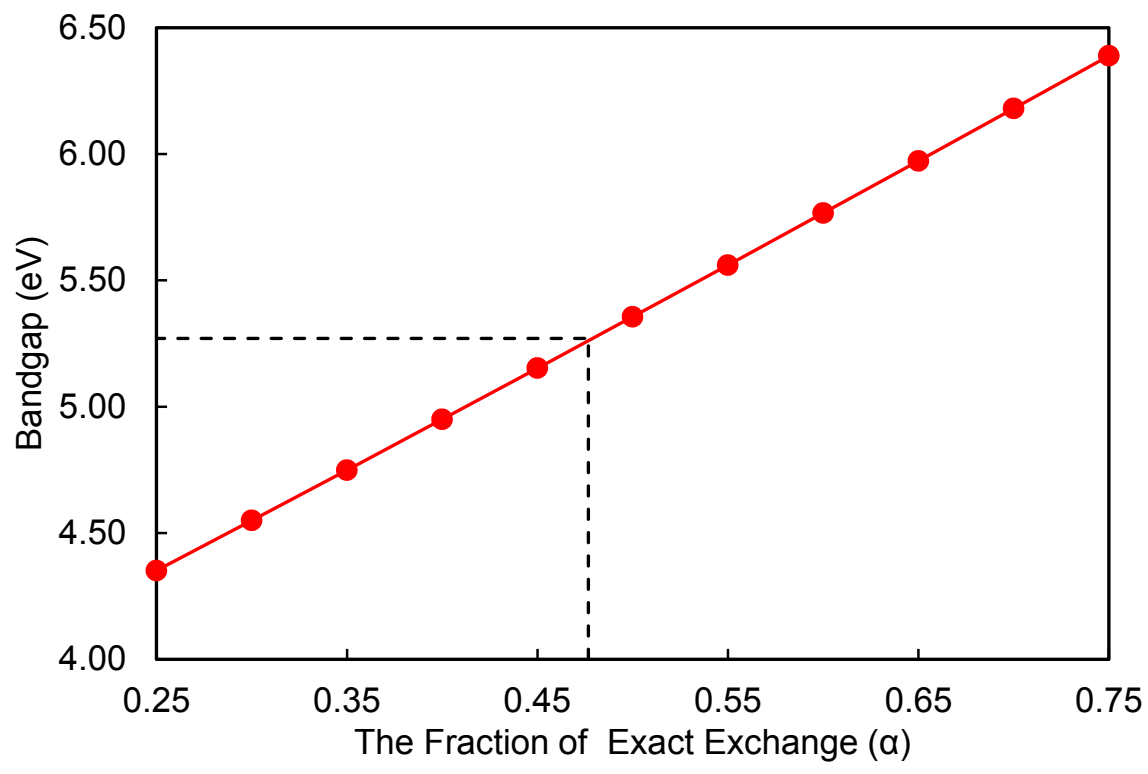

Figure S1. Bandgap of $\mathrm{Li}_{2} \mathrm{~S}$ calculated by the Heyd-Scuseria-Ernzerhof hybrid functional with various fractions of exact exchange $(\alpha)$. 


\section{Details of the coupling constant and adiabadicity correction calculations}

In equation 5 of the main article an adiabadicity correction $\Delta$ is added to the standard MarcusLandau-Zener equation. It is given by ${ }^{1}$

$$
\Delta=\left|H_{a b}\right|+\frac{\lambda}{2}-\sqrt{\frac{\lambda^{2}}{4}+\left|H_{a b}\right|^{2}}
$$

The electronic coupling constant $H_{a b}$ is computed using the procedure of Van Voorhis. ${ }^{2-3}$ First, it is noticed that the constrained DFT (cDFT) states are eigenstates of a modified Hamiltonian

$$
\left(H_{c}^{K S}+V_{c} w_{c}\right) \Psi_{c}=F_{c} \Psi_{c}
$$

with $F_{c}=\left\langle\Psi_{c}\left|H+V_{c} w_{c}\right| \Psi_{c}\right\rangle$. Then the coupling constant $H_{a b}=\left\langle\Psi_{a}|H| \Psi_{b}\right\rangle$ can be written as

$$
H^{\prime}{ }_{a b}=F_{b}\left\langle\Psi_{a} \mid \Psi_{b}\right\rangle-V_{c}^{b}\left\langle\Psi_{a}\left|w_{c}^{b}(r)\right| \Psi_{b}\right\rangle
$$

Where $\left\langle\Psi_{a} \mid \Psi_{b}\right\rangle=S_{a b}$ is the overlap element and $V_{c}^{b}\left\langle\Psi_{a}\left|w_{c}^{b}\right| \Psi_{b}\right\rangle=W_{a b}$ is the weight element. Working in the basis of two nonorthogonal cDFT states leads to the following overlap matrix

$$
\boldsymbol{S}=\left(\begin{array}{cc}
1 & S_{a b} \\
S_{b a} & 1
\end{array}\right)
$$

and Hamiltonian matrix

$$
\boldsymbol{H}^{\prime}=\left(\begin{array}{cc}
E_{a}^{K S} & H^{\prime}{ }_{a b} \\
H^{\prime}{ }_{b a} & E_{b}^{K S}
\end{array}\right)
$$

cDFT states are nonorthogonal; however, the Hamiltonian matrix needs to be presented in an orthogonal basis. This is done using Löwdin orthogonalization

$$
H=S^{-1 / 2} H^{\prime} S^{-1 / 2}
$$

The coupling constants given in the main article are the off-diagonal elements of $\boldsymbol{H}$. To check the correctness of the coupling constants, they were computed also using the overlap method of Migliore. ${ }^{4-5}$ In this approach the ground state DFT (not cDFT) wave function is written as a linear combination of the two constrained DFT states

$$
\left|\Psi_{G S}\right\rangle=a\left|\Psi_{a}\right\rangle+b\left|\Psi_{b}\right\rangle
$$

from which

$$
A \equiv\left\langle\Psi_{a} \mid \Psi_{G S}\right\rangle=a+b\left\langle\Psi_{a} \mid \Psi_{b}\right\rangle \text { and } B \equiv\left\langle\Psi_{b} \mid \Psi_{G S}\right\rangle=b+a\left\langle\Psi_{b} \mid \Psi_{a}\right\rangle
$$

After going through the detailed derivation in the references above, the following equation is obtained for the coupling constants

$$
H_{a b}=\left|\left(\frac{A B}{A^{2}-B^{2}}\right) \Delta E_{a b}\left(1-\frac{A^{2}+B^{2}}{2 A B}\right)\left(\frac{1}{1-\left(\left\langle\Psi_{a} \mid \Psi_{b}\right\rangle\right)^{2}}\right)\right|
$$

Where $\Delta E_{a b}(\boldsymbol{R})=(\boldsymbol{R})-E_{b}^{K S}(\boldsymbol{R})$ and the dependency on the nuclear coordinates $\boldsymbol{R}$ is stressed.

The coupling constants computed using either Eq. S6 or Eq. S9 are in good agreement and do not differ by more than $20 \%$.

The needed overlap and weight elements were calculated using all-electron PAW-cDFT states as detailed in Ref. 6 using an interpolation grid density of $0.04 \AA$. 


\section{Ehrenfest dynamics of hole transfer in $\alpha-S$}

Based on the results obtained in the main portion of the manuscript, it is highly unlikely that holes are transferred by a polaronic hopping mechanism in $\alpha$-S. Instead, experimental conductivity studies suggest that band conduction prevails. To understand the hole transfer in sulfur without $a$ priori assumptions about the mechanism, coupled electron-nuclear quantum dynamics within the Ehrenfest framework were performed. Ehrenfest dynamics is a semi-classical approach to quantum dynamics in which the electron motion is obtained from the time-dependent Schrödinger equation while nuclei move according to classical mechanics on a single, mean-field potential energy surface. Within DFT, the electron density can be propagated using real-time time-dependent DFT while the nuclei follow the average potential energy surface:

$$
\begin{gathered}
i \frac{\partial \psi}{\partial t}=\widehat{H}_{e l}(\boldsymbol{r}, \boldsymbol{R}) \psi(t, \boldsymbol{r} ; \boldsymbol{R}) \\
M_{A} \frac{\partial^{2} \boldsymbol{R}_{A}}{\partial t^{2}}=-\nabla_{\boldsymbol{R}_{A}}\left\langle\psi\left|\widehat{H}_{e l}\right| \psi\right\rangle
\end{gathered}
$$

Where $\psi$ is the wave function depending explicitly on time $t$ and positions of the electrons $\boldsymbol{r}$ as well as implicitly on nuclear positions $\boldsymbol{R}$. In the present work, these equations are solved within the PAW approach as implemented in GPAW. ${ }^{7}$ The nuclei are propagated using the velocity Verlet algorithms while electrons are propagated with the semi-implicit Crank-Nicholson method (for details, see the GPAW documentation). For both a time-step of 15 attoseconds is used. The gridspacing was set to $0.16 \AA$ and exchange-correlation effects were accounted using the PBE functional. The unit cell was the same as used for sulfur in the main article.

The Ehrenfest dynamics were initiated from a cDFT localized state where the hole is constrained to exist within a single $\mathrm{S}_{8}$ ring. A short, $3 \mathrm{ps}$ cDFT molecular-dynamics at $300 \mathrm{~K}$ was performed before initializing the Ehrenfest dynamics in order to thermalize the system. During the Ehrenfest simulation the spin density of the systems was followed and the results are shown in Figure S2.

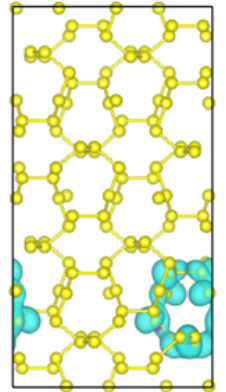

$0 \mathrm{fs}$

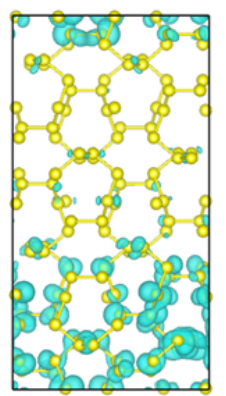

$4.5 \mathrm{fs}$

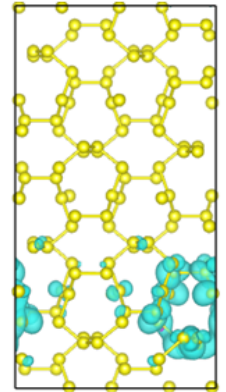

0.75 fs

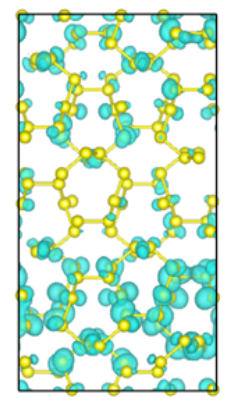

$7.5 \mathrm{fs}$

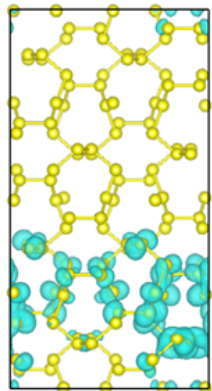

$1.5 \mathrm{fs}$

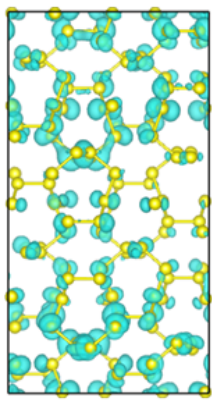

$10.5 \mathrm{fs}$

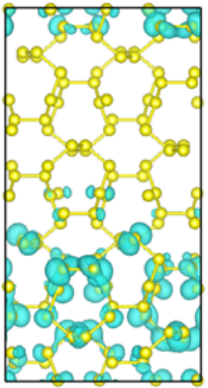

$3.0 \mathrm{fs}$ 


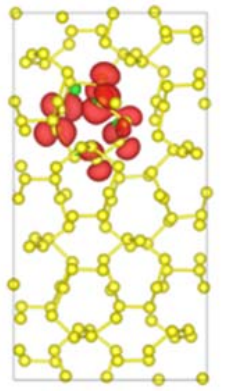

$0 \mathrm{fs}$

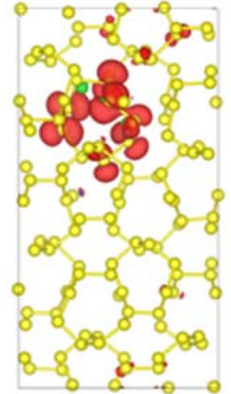

$5 \mathrm{fs}$

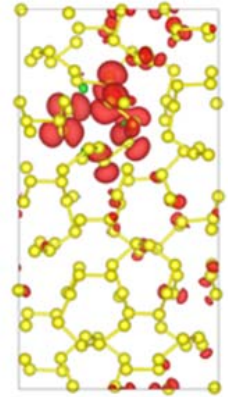

$7 \mathrm{fs}$

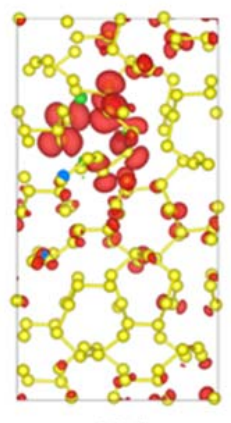

$10 \mathrm{fs}$

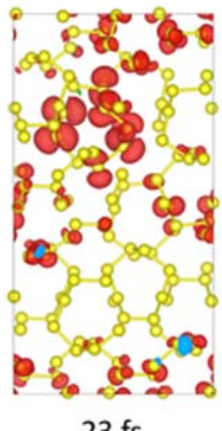

$23 \mathrm{fs}$

Figure S2. Evolution of the hole spin-density during Ehrenfest dynamics. Top: Hole in $\mathrm{S}_{8}$. The cyan isosurface is the spin density difference recorded at different times (indicated in the figure) during the simulation. Bottom: Hole in $\mathrm{S}_{9}$. The red isosurface is the spin density difference recorded at different times (indicated in the figure) during the simulation.

From Figure S2, it can be seen that in the beginning of the Ehrenfest dynamics, the hole is localized on a single $\mathrm{S}_{8}$ or $\mathrm{S}_{9}$ sulfur ring. After 1.5 femtoseconds, the initially localized hole on a $\mathrm{S}_{8}$ ring is already delocalized on four $S_{8}$ rings; within 10.5 femtoseconds the hole is delocalized over the entire simulation cell. The structural changes during the delocalizing are minimal, under 0.01 $\AA$. Based on visual inspection, the delocalized hole is on p-type orbitals. Given the fast delocalization and minimal changes in the bond lengths, it is highly unlikely that hole transport in pure $\alpha$-sulfur is mediated by polaronic hopping. Instead, the p-type character of the delocalized hole, combined with its fast delocalization dynamics without geometric changes, is a strong indication that holes in sulfur are transferred by a p-type band conduction mechanism.

In contrast, hole polarons that are initially localized on a $\mathrm{S}_{9}$ defect ring appear to be meta-stable. As seen from the bottom panel of Fig. S2, these holes are localized on the ring for up to $5 \mathrm{fs}$. Even after $23 \mathrm{fs}$ the hole remains primarily localized on this ring, with significant changes in the S-S bonds (up to $0.3 \AA$ ) occurring during the Ehrenfest dynamics. We therefore conclude that $S_{9}$ rings can act as polaron traps. 
Table S1. Calculated diffusivity at room Temperature $\left(\mathrm{k}_{\mathrm{b}} \mathrm{T}=0.0256 \mathrm{eV}\right)$. Prior calculations on $\mathrm{Li}_{2} \mathrm{O}_{2}(\mathrm{Ref}$ 8) and $\mathrm{Na}_{2} \mathrm{O}_{2}$ (Ref. 9) are also shown for comparison.

\begin{tabular}{|c|c|}
\hline Defect Type & $\mathrm{D}\left(\mathrm{cm}^{2} / \mathrm{s}\right)$ \\
\hline \multicolumn{2}{|c|}{$\alpha-S$} \\
\hline p- (Intra-ring) & $8 \times 10^{-5}$ \\
\hline p- (Inter-ring) & $5 \times 10^{-9}$ \\
\hline $\mathrm{p}+(\text { Intra-ring })^{\dagger}$ & --- \\
\hline $\mathrm{p}+(\text { Inter-ring })^{\dagger}$ & --- \\
\hline Delocalized hole & $3 \times 10^{-2}-3 \times 10^{-1+}$ \\
\hline $\mathrm{S}_{7}^{-}$ & $8 \times 10^{-12}$ \\
\hline $\mathrm{S}_{9}^{+}$ & $8 \times 10^{-30}$ \\
\hline $\mathrm{S}_{6}^{+}$ & --- \\
\hline $\mathrm{S}_{7}^{+}$ & $8 \times 10^{-23}$ \\
\hline \multicolumn{2}{|c|}{$\mathrm{Li}_{2} \mathrm{~S}$} \\
\hline $\mathrm{p}^{+}$ & $8 \times 10^{-4}$ \\
\hline p- & --- \\
\hline $\mathrm{V}_{\mathrm{Li}}^{-}$ & $3 \times 10^{-8}$ \\
\hline $\mathrm{Li}^{+}$ & $3 \times 10^{-11}$ \\
\hline $\mathrm{F}_{\mathrm{Li}}$ & --- \\
\hline $\mathrm{F}_{\mathrm{S}}$ & --- \\
\hline Sch & --- \\
\hline \multicolumn{2}{|c|}{$\mathrm{Li}_{2} \mathrm{O}_{2}$} \\
\hline $\mathrm{p}^{+}$ & --- \\
\hline $\mathrm{V}_{\mathrm{Li}}^{-}$ & $2 \times 10^{-10 *}$ \\
\hline \multicolumn{2}{|c|}{$\mathrm{Na}_{2} \mathrm{O}_{2}$} \\
\hline $\mathrm{p}^{+}$ & --- \\
\hline $\mathrm{V}_{\mathrm{Na}}{ }^{-}$ & $2 \times 10^{-11 *}$ \\
\hline
\end{tabular}

${ }^{*}$ Calculated from published activation energy $\left(\mathrm{E}_{\mathrm{b}}\right)$ assuming room temperature (300 $\left.\mathrm{K}\right)$ and an attempt frequency of $10^{13} \mathrm{~s}^{-1}$ (Ref. 8 and Ref. 9). ${ }^{\dagger}$ The hole polaron in $\alpha-S$ is kinetically unstable and thus delocalized. ${ }^{\ddagger}$ The experimental mobility data is taken from Ref.1o, and room temperature is used to calculate the diffusivity. 
Figure S3. (a) and (b): Magnetization density isosurfaces for metastable hole polarons in $\mathrm{Li}_{2} \mathrm{~S}$. (c) and (d): contour plots of the magnetization density on a (110) plane. Red and blue areas represent magnetization densities of $0.06 \mathrm{e} / \mathrm{bohr}^{3}$ and o e/bohr ${ }^{3}$, respectively. Energies given at the bottom represent the relative energy of these polarons with respect to that of the lowest energy polaron configuration discussed in the main text. Blue and yellow spheres represent Li and S atoms, respectively. Dotted white lines represent the symmetry-identical orientations of the lowest-energy hole polaron configuration.

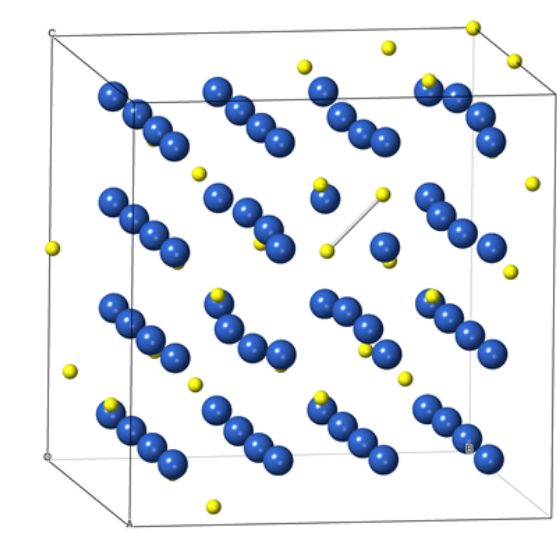

(a) $\mathrm{S}_{2}{ }^{2-}$ formed in the presence of $2 \mathrm{~V}_{\mathrm{Li}}{ }^{0}$

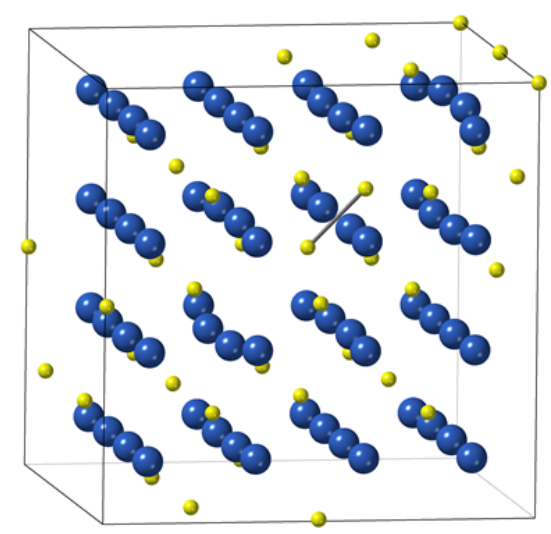

(b) $\mathrm{S}_{2}{ }^{2-}$ formed from the combination of $2 \mathrm{p}^{+}$

Figure $\mathrm{S}_{4}$. Structure of the $\mathrm{Li}_{2} \mathrm{~S}$ supercell containing $\mathrm{S}_{2}{ }^{2-}$ dimers formed from the insertion of: (a) two neutral $\mathrm{Li}$ vacancies, or (b) two hole polarons. Blue and yellow spheres represent $\mathrm{Li}$ and $\mathrm{S}$ atoms, respectively. 


\section{Analysis of the hole and electron polaron traps in $\alpha-S$.}

We have performed an analysis of the alignment of the hole (electron) polaron states relative to the top (bottom) of the valence (conduction) band in the different $S_{x}(x=6-10)$ rings in $\alpha-S$. The analysis is based on the formation energies of the neutral, positively, and negatively charged rings. These formation energies are shown in Table 1 of the main text and repeated in Table S2 for convenience. The structures of the neutral $S_{x}$ rings are shown in Figure $S_{5}$.

By subtracting the formation energy of the positively (negatively) charged $S_{x}$ ring from the formation energy of the neutral ring it is possible to obtain the energy for a localized hole (electron) polaron for that ring configuration. Comparing those energies to the formation energies of the delocalized holes (electrons), which are also shown in Table S2, allows us to determine the energy position of a localized hole (electron) polaron relative to the top (bottom) of the valence (conduction) band. These data are shown in Table S2 and Figure S6.

It can be concluded that hole polarons are only stable in $S_{9}$ and $S_{10}$ rings, implying that these rings can act as hole polaron traps. In contrast, electron polarons are stable in all the studied $S_{x}$ rings. Furthermore, electron polarons are most stable in $\mathrm{S}_{7}$ rings, which are $0.38 \mathrm{eV}$ more stable than in $\mathrm{S}_{8}$ rings, Figure $\mathrm{S} 6$. This implies that the electrons will be transported by means of polaronic hopping between $\mathrm{S}_{8}$ rings, with $\mathrm{S}_{7}$ rings as traps.

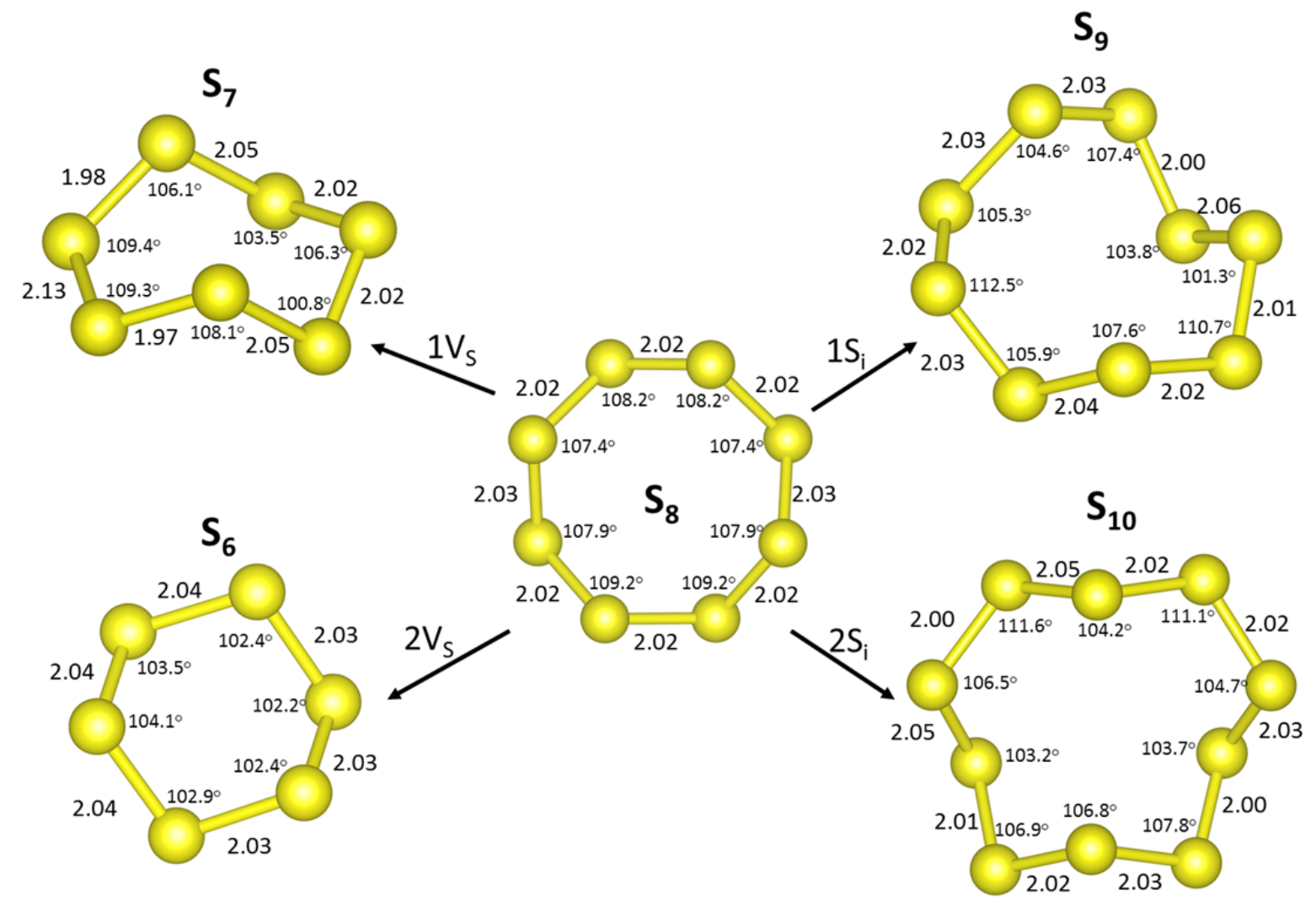

Figure $S_{5}$. Calculated structures of various neutral $S_{x}\left(x=6\right.$ to10) rings in $\alpha-S$. The $S_{8}$ ring is the expected molecular unit in the defect-free crystal structure. $S_{6}$ and $S_{7}$ correspond to ring structures formed upon the addition of 1 or 2 vacancies, respectively. Similarly, $S_{9}$ and $S_{10}$ result from the addition of 1 or 2 interstitials. 
Table S2. Formation energies, $E_{f}$, of various charged and neutral $S_{x}$ rings $(x=6-10)$ in in $\alpha-S$, and stabilization energies of hole and electron polarons compared to the delocalized states. All energies are in $\mathrm{eV}$.

\begin{tabular}{cccccc}
\hline Defect Type & $\begin{array}{c}E_{f} \\
\text { Neutral } \\
\text { Defect }\end{array}$ & $\begin{array}{c}E_{f} \\
\text { Positive } \\
\text { Defect }\end{array}$ & $\begin{array}{c}E_{f} \\
\text { Negative } \\
\text { Defect }\end{array}$ & $\begin{array}{c}\text { Energy of } \mathrm{h}^{+} \\
\text {relative to } \\
\text { delocalized } \mathrm{h}^{+} \text {in } \alpha- \\
\mathrm{S}^{\mathrm{a}}\end{array}$ & $\begin{array}{c}\text { Energy of e relative to } \\
\text { delocalized } \mathrm{e}^{-} \text {in } \alpha-\mathrm{S}^{\mathrm{a}}\end{array}$ \\
\hline $\mathrm{S}_{6}$ & 0.18 & 2.22 & 2.84 & 0.08 & -0.12 \\
$\mathrm{~S}_{7}$ & 0.25 & 2.24 & 2.23 & 0.03 & -0.80 \\
$\mathrm{~S}_{8}$ & - & 2.44 & 2.36 & 0.48 & -0.42 \\
$\mathrm{~S}_{9}$ & 0.56 & 2.22 & 3.33 & -0.30 & -0.01 \\
$\mathrm{~S}_{10}$ & 1.03 & 2.63 & 3.48 & -0.36 & -0.33 \\
$\begin{array}{c}\text { Delocalized } \\
\text { electrons/holes }\end{array}$ & - & $1.96^{\mathrm{b}}$ & $2.78^{\mathrm{b}}$ & - & - \\
\hline
\end{tabular}

${ }^{a}$ The energies are calculated as previously described in the present Supplementary Material. ${ }^{b}$ Evaluated for delocalized holes as the difference: $\left[\mathrm{E}_{f}\left(\mathrm{~S}_{8}{ }^{+}\right)-\mathrm{h}^{+}\right.$stabilization energy], and similarly for the delocalized electron, $\left[\mathrm{E}_{\mathrm{f}}\left(\mathrm{S}_{8}^{-}\right)-\mathrm{e}^{-}\right.$stabilization energy].
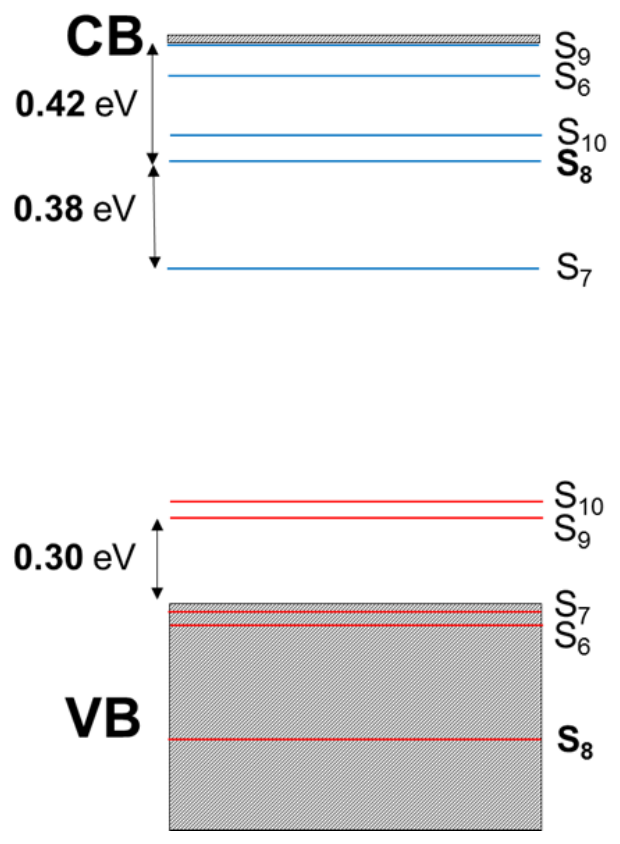

Figure S6. Schematic of the estimated energy levels of hole (red lines) and electron (blue lines) polarons in different $S_{x}$ rings in $\alpha-S$ with respect to the valence (VB) and conduction (CB) bands. Hole polarons occupying the $S_{6}, S_{7}$, and $S_{8}$ rings have energies that lie within the $\mathrm{VB}$, and thus will delocalize into band-like states.

\section{REFERENCES}

1. Blumberger, J.; McKenna, K. P., Constrained density functional theory applied to electron tunnelling between defects in MgO. Phys. Chem. Chem. Phys. 2013, 15 (6), 2184-2196. 
2.Kaduk, B.; Kowalczyk, T.; Van Voorhis, T., Constrained Density Functional Theory. Chemical Reviews 2012, 112 (1), 321-370.

3.Wu, Q.; Voorhis, T. V., Extracting electron transfer coupling elements from constrained density functional theory. J. Chem. Phys. 20o6, 125 (16), 164105.

4.Migliore, A., Nonorthogonality Problem and Effective Electronic Coupling Calculation: Application to Charge Transfer in $\pi$-Stacks Relevant to Biochemistry and Molecular Electronics. J. Chem. Theory Comput. 2011, 7 (6), 17121725 .

5.Migliore, A., Full-electron calculation of effective electronic couplings and excitation energies of charge transfer states: Application to hole transfer in DNA $\pi$-stacks. J. Chem. Phys. 2009, 131 (11), 114113.

6.Melander, M.; Jónsson, E. O.; Mortensen, J. J.; Vegge, T.; García Lastra, J. M., Implementation of Constrained DFT for Computing Charge Transfer Rates within the Projector Augmented Wave Method. J. Chem. Theory Comput. 2016, 12 (11), 5367-5378.

7.Ojanperä, A.; Havu, V.; Lehtovaara, L.; Puska, M., Nonadiabatic Ehrenfest molecular dynamics within the projector augmented-wave method. J. Chem. Phys. 2012, 136 (14), 144103.

8.Radin, M. D.; Siegel, D. J., Charge transport in lithium peroxide: relevance for rechargeable metal-air batteries. Energy Environ. Sci. 2013, 6 (8), 2370-2379.

9. Yang, S.; Siegel, D. J., Intrinsic Conductivity in Sodium-Air Battery Discharge Phases: Sodium Superoxide vs Sodium Peroxide. Chem. Mater. 2015, 27 (11), 3852-386o.

10. Gill, W. D.; Street, G. B.; Macdonald, R. E., Transport properties of vapor grown orthorhombic sulfur crystals. J. Phys. Chem. Solids 1967, 28 (8), 1517-1518. 